\title{
The effect of the enhanced recovery after surgery program on lung cancer surgery: a systematic review and meta-analysis
}

\author{
Rongyang $\mathrm{Li}^{1,2}$, Kun Wang ${ }^{1,2}$, Chenghao $\mathrm{Qu}^{1,2}$, Weifeng $\mathrm{Qi}^{1,2}$, Tao Fang ${ }^{1,2}$, Weiming Yue ${ }^{1,2}$, Hui Tian ${ }^{1,2}$ \\ ${ }^{1}$ Department of Thoracic Surgery, Qilu Hospital, Cheeloo College of Medicine, Shandong University, Jinan, China; ${ }^{2}$ Cheeloo College of Medicine, \\ Shandong University, Jinan, China \\ Contributions: (I) Conception and design: R Li, H Tian; (II) Administrative support: H Tian, W Yue, C Qu; (III) Provision of study materials or \\ patients: R Li, K Wang; (IV) Collection and assembly of data: R Li, K Wang, W Qi, T Fang; (V) Data analysis and interpretation: R Li, K Wang, C \\ $\mathrm{Qu}$; (VI) Manuscript writing: All authors; (VII) Final approval of manuscript: All authors. \\ Correspondence to: Hui Tian. Department of Thoracic Surgery, Qilu Hospital, Cheeloo College of Medicine, Shandong University, Jinan 250012, \\ China. Email: tianhuiq1@126.com.
}

Background: Lung cancer is one of the most common causes of cancer-related death worldwide. The enhanced recovery after surgery (ERAS) program is an effective evidence-based multidisciplinary protocol of perioperative care. However, the roles of ERAS in lung cancer surgery remain unclear. This systematic review and meta-analysis aimed to investigate the short-term impact of the ERAS program on lung resection surgery, especially in relation to postoperative complications.

Methods: A systematic literature search of PubMed, EMBASE, and the Cochrane Library databases until October 2020 was performed to identify the studies that implemented an ERAS program in lung cancer surgery. The studies were selected and subjected to data extraction by 2 reviewers independently, which was followed by quality assessment. A random effects model was used to calculate overall effect sizes. Risk ratio (RR), risk difference (RD), and standardized mean difference (SMD) with $95 \%$ confidence interval (CI) served as the summary statistics for meta-analysis. Subgroup analysis and sensitivity analysis were subsequently performed.

Results: A total of 21 studies with 6,480 patients were included. The meta-analysis indicated that patients in the ERAS group had a significantly reduced risk of postoperative complications ( $\mathrm{RR}=0.64$; $95 \%$ CI: 0.52 to 0.78 ) and shortened postoperative length of stay (SMD=-1.58; 95\% CI: -2.38 to -0.79 ) with a significant heterogeneity. Subgroup analysis showed that the risks of pulmonary (RR $=0.58 ; 95 \% \mathrm{CI}: 0.45$ to 0.75 ), cardiovascular ( $\mathrm{RR}=0.73 ; 95 \% \mathrm{CI}: 0.59$ to 0.89$)$, urinary ( $\mathrm{RR}=0.53 ; 95 \% \mathrm{CI}: 0.32$ to 0.88$)$, and surgical complications ( $\mathrm{RR}=0.64 ; 95 \% \mathrm{CI}$ : 0.42 to 0.97$)$ were significantly lower in the ERAS group. No significant reduction was found in the in-hospital mortality $(\mathrm{RD}=0.00 ; 95 \% \mathrm{CI}:-0.01$ to 0.00 ) and readmission rate $(\mathrm{RR}=1.00 ; 95 \% \mathrm{CI}: 0.76$ to 1.32 ). In the qualitative review, most of the evidence reported significantly decreased hospitalization costs in the ERAS group.

Conclusions: The implementation of an ERAS program for surgery of lung cancer can effectively reduce risks of postoperative complications, length of stay, and costs of patients who have undergone lung cancer surgery without compromising their safety.

Keywords: Enhanced recovery after surgery (ERAS); lung cancer surgery; systematic review; meta-analysis

Submitted Mar 13, 2021. Accepted for publication May 14, 2021.

doi: $10.21037 /$ jtd-21-433

View this article at: https://dx.doi.org/10.21037/jtd-21-433

(c) Journal of Thoracic Disease. All rights reserved. 


\section{Introduction}

Lung cancer is one of the most common causes of cancerrelated death worldwide $(1,2)$, the most common cancer and the leading cause of cancer death in men. Meanwhile, it is the third most common cancer in women (after breast and colorectal cancer) and the second leading cause of cancer death (after breast cancer) $(3,4)$. Surgical resection remains the most preferable treatment for early-stage lung cancer even for those patients with advanced cancer (1). With the implementation of minimally invasive techniques, the preferred surgical method has gradually developed from thoracotomy to video-assisted thoracoscopic surgery (VATS) $(5,6)$. Although VATS is becoming increasingly popular in clinical practice, some important side effects and postoperative complications are still inevitable, such as pneumonia, pleural effusion, pneumothorax, arrhythmia, respiratory failure, and others (7-9). The occurrence of postoperative complications is related to many perioperative factors, such as poor preoperative lung function, improper operation by the surgeon, inadequate expectoration after surgery, sputum accumulation, and long duration of chest tube placement. Therefore, it is urgent to optimize perioperative management in order to reduce the occurrence of complications and improve the quality of life (QoL) of patients.

The Enhanced Recovery After Surgery (ERAS) program, which has become prevalent in recent years, is a multimodal approach encompassing all phases of care: preoperative (including assessment, counselling, nutrition, and smoking cessation), intraoperative (including minimally invasive surgery, standardized anesthetic protocol, and single chest tube placement), and postoperative (including early ambulation, nutrition, and adequate pain relief) $(10,11)$. It is a combination of various procedures spanning patient admission through to discharge and is aimed at minimizing surgical stress, reducing the occurrence of postoperative complications, decreasing length of stay (LOS), increasing QoL during hospitalization, and thus reducing costs related to surgery. The concept of ERAS was first proposed by Dr. Engelman in 1994 (12). In recent years, this multidisciplinary and multimodal perioperative rehabilitation concept has been widely applied in surgeries of various disciplines: colorectal (13), liver (14), gynecological (15), urologic (16), spinal (17), and gastrointestinal surgery (18). Moreover, recent studies have indicated that patients treated with the ERAS program during hepatectomy, laparoscopic colorectal surgery, and spinal surgery have a significant reduction in LOS and risk of postoperative complications $(13,14,17)$. Although ERAS has been used in lung cancer surgery for several years, its effectiveness is still controversial.

A recent systematic review and meta-analysis of 38 randomized controlled trials (RCTs) found that the ERAS program plays an important role in reducing the rate of complications and shortening the LOS across various surgical specialties (19). However, the findings of this study may not be applicable to pulmonary resection owing to the limited data. Fiore et al. performed a systematic review and meta-analysis in order to evaluate the efficacy of the ERAS program in elective lung resection in 2016 (20), which included 6 studies (1 RCT, 1 case-control, 4 cohort); however, the authors finally failed to reach a convincing conclusion because of the small sample size and high bias risks. A later evidenced-based review and meta-analysis reported by Li et al. in 2017 (21) that included 7 RCTs, demonstrated that the ERAS program in lung cancer surgery could effectively accelerate postoperative recovery and save hospitalization costs without compromising patient safety; however, the inappropriate inclusion criteria and relatively small sample size might have produced considerable biases and reduced the reliability of their results. In order to reach a more substantiated conclusion, we aimed to conduct a systematic review and metaanalysis to determine the short-term impact of the ERAS program on lung resection surgery, especially in relation to postoperative complications.

We present the following article in accordance with the PRISMA reporting checklist (available at https://dx.doi. org/10.21037/jtd-21-433).

\section{Methods}

This systematic review and meta-analysis was conducted and reported in accordance with the Meta-Analysis of Observational Studies in Epidemiology (MOOSE) guidelines (22) and the PRISMA statement (23). The review was registered at the International Prospective Register of Systematic Reviews (PROSPERO; https:// www.crd.york.ac.uk/PROSPERO/) as registration number CRD42020216064.

\section{Databases and search strategy}

The literature review was performed based on 3 online databases: PubMed, EMBASE, and the Cochrane Library 
Table 1 Details of search strategy for all databases

\begin{tabular}{ll}
\hline Database & Search Strategy \\
\hline PubMed & $((((((($ Enhanced[All Fields] AND recovery[All Fields]) OR Fast-track[All Fields]) OR ((intensive[All Fields] OR \\
& intensity[All Fields]) AND (rehabilitation[Subheading] OR rehabilitation[All Fields] OR rehabilitation[MeSH Terms]))) \\
& OR (accelerated[All Fields] AND (rehabilitation[Subheading] OR rehabilitation[All Fields] OR rehabilitation[MeSH \\
& Terms]))) OR ERAS[All Fields]))) AND ((((((((((((()(((Pulmonary Neoplasms) OR Neoplasms, Lung) OR Lung \\
& Neoplasm) OR Neoplasm, Lung) OR Neoplasms, Pulmonary) OR Neoplasm, Pulmonary) OR Pulmonary Neoplasm) \\
& OR Lung Cancer) OR Cancer, Lung) OR Cancers, Lung) OR Lung Cancers) OR Pulmonary Cancer) OR Cancer, \\
& Pulmonary) OR Cancers, Pulmonary) OR Pulmonary Cancers) OR Cancer of the Lung) OR Cancer of lung)) OR \\
& "Lung Neoplasms"[Mesh]) \\
& ('cancer of lung' OR 'cancer of the lung' OR 'pulmonary cancers' OR 'pulmonary cancer' OR 'lung cancer' OR \\
& 'lung cancers' OR 'pulmonary neoplasm' OR 'lung neoplasm' OR 'pulmonary neoplasms' OR 'lung cancer'/exp) \\
& AND ('enhanced recovery' OR 'fast track' OR 'intensive rehabilitation' OR 'accelerated rehabilitation' OR eras) \\
& $(($ LUNG NEOPLASM[MeSH]) OR ((LUNG OR PULMON*) AND (NEOPLAS* OR CANCER OR CARCINOMA*))) AND \\
& $(($ enhanced recovery) OR (fast track) OR (intensive rehabilitation) OR (accelerated rehabilitation) OR ERAS) \\
\hline
\end{tabular}

(from 29 September 2020 to 3 October 2020). The Medical Subject Headings (MeSH) included in the search strategy were "lung neoplasms" and "rehabilitation", and the free terms were searched in PubMed. The key terms and free terms were used in every possible combination by 2 Boolean operators ("AND" and "OR"). The details of search strategies for all databases are displayed in Table 1. The articles were independently reviewed and cross-checked by 2 authors (Rongyang Li and Kun Wang). Moreover, we also manually searched the reference lists of excluded publications to identify any further potential nonduplicate studies. Any disagreements among the reviewers were resolved by discussion.

\section{Study selection and criteria}

The inclusion criteria were as follows: (I) involved adult patients undergoing elective pulmonary resection (wedge resection, lobectomy, pneumonectomy, etc.), (II) involved patients who received an ERAS program (we recognized a total of 25 elements in studies encompassing all phases of perioperative care [pre-, intra-, and postoperative] (20,21,24,25), as shown in Figure 1), (III) involved an ERAS program with at least 4 elements that covered at least 2 phases of perioperative care, (IV) involved a traditional care control group that had adopted at least 3 elements fewer than the ERAS group, (V) reported at least 1 of the outcome measures of interests (see below), and (VI) written in English.

The exclusion criteria were as follows: (I) ineligible article types, such as case report, reviews, and conference abstracts; (II) no outcomes of interest present; (III) inclusion of nonhuman participants or written in languages other than English.

\section{Endpoints and outcome measures}

The primary outcome was postoperative complication, which was defined by the presence of any individual complication during the hospitalization or within 30 days after surgery. Other outcomes of interest were in-hospital mortality, LOS, 30-day readmission rate, and total cost of hospitalization.

\section{Data collection}

The 2 reviewers (Rongyang Li and Kun Wang) independently browsed the eligible studies and extracted the corresponding data to fill in a predefined table. Any discrepancies were resolved by consensus. The following data were extracted from each study: (I) publication data: authors, year, and country; (II) experimental data: study design, study period, ERAS elements, surgical procedures, and extent of resection; (III) demographic data: sample size, age, gender, body mass index (BMI), and predicted forced expiratory volume in 1 second (FEV1\%); (IV) outcome data: postoperative complications, in-hospital mortality, LOS, 30-day readmission, and total cost of hospitalization. We did not contact authors to obtain any unpublished data.

\section{Quality assessment}

Quality of included cohort studies was evaluated using the 


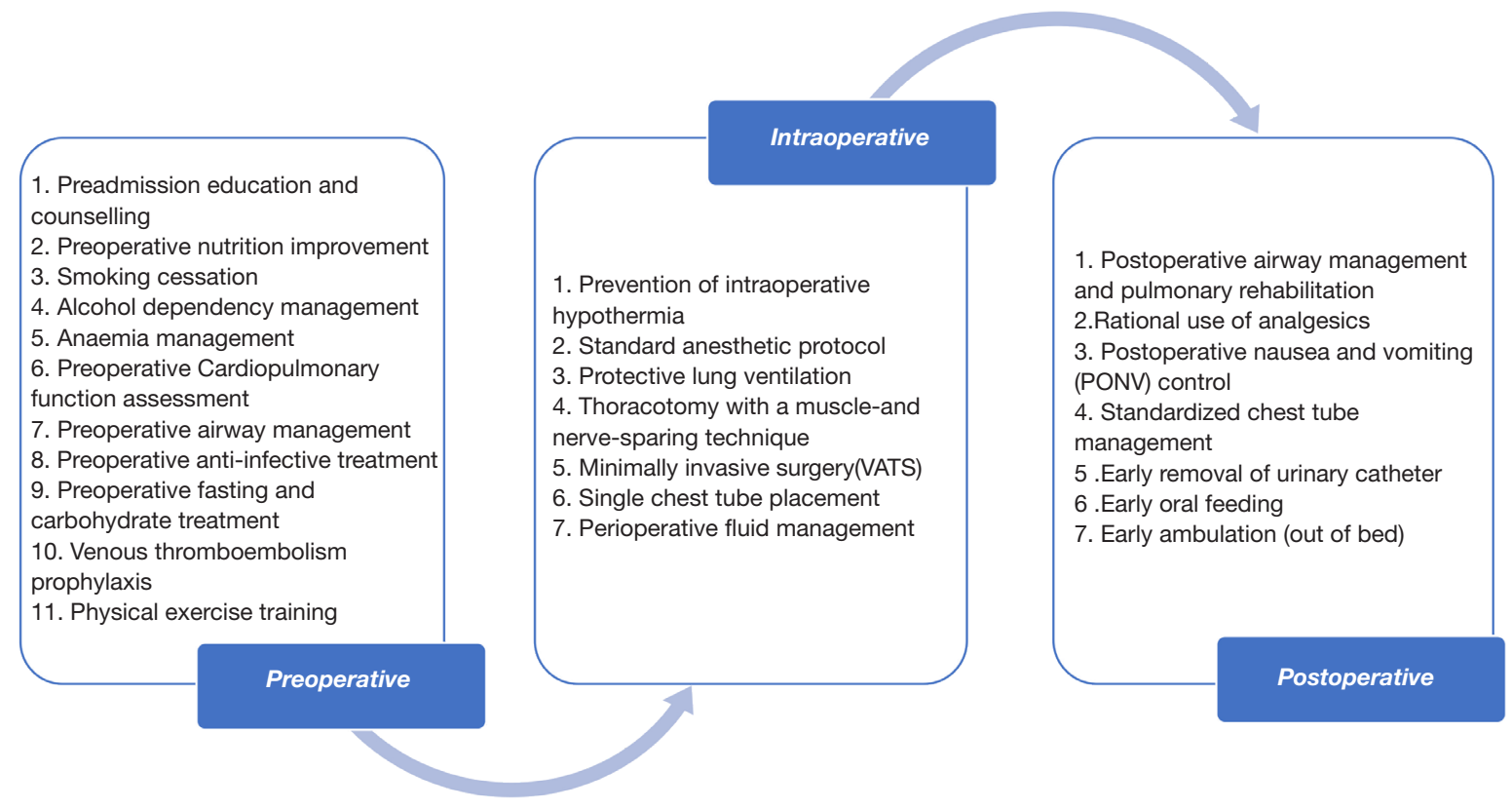

Figure 1 Care elements implemented in the ERAS program for lung cancer surgery. ERAS, enhanced recovery after surgery; VATS, videoassisted thoracoscopic surgery; PONV, postoperative nausea and vomiting.

Newcastle-Ottawa Quality Assessment Scale (NOS) (26). We identified studies with a score equal to or higher than 6 as eligible for our meta-analysis. We used the Cochrane risk of bias tool to assess the quality of RCTs (27). Due to the nature of the interventions involved in ERAS, it is often unfeasible to blind patients and staff; therefore, high risk of performance bias was assumed if a study did not mention blinding of staff or patients.

The quality of each study was independently appraised by 2 investigators (Rongyang Li and Kun Wang). Any disagreements regarding the quality assessment were resolved by consulting a third investigator (Chenghao $\mathrm{Qu}$ ).

\section{Statistical analysis}

We calculated the risk ratio (RR) with $95 \%$ confidence interval (CI) to summarize the effects of ERAS programs on dichotomous data. If no events occurred in both the experimental and control group, the pooled risk difference (RD) estimates and 95\% CIs were applied. The standardized mean difference (SMD) with $95 \%$ CI served as the appropriate statistics to summarize the mean values with standard deviations (SDs) for continuous variables. If the SDs were not provided, we did not incorporate the data in the quantitative synthesis because the extrapolation of SDs was only applicable for studies with a large sample size and normal distribution of outcomes according to the guidelines of the Cochrane Collaboration (27). Meta-analysis was not performed when the number of studies was very small $(n<5)$, because the results might have been misleading; instead, a qualitative summary of evidence was performed.

Cochrane Q test and $\mathrm{I}^{2}$ statistics were used to quantify the heterogeneity level. An $\mathrm{I}^{2}$ of $25 \%, 50 \%$, and $75 \%$ represented low, moderate, and considerable variance, respectively (28). A 2 -sided $\mathrm{P}$ value of less than 0.05 was defined as statistical significance. In our study, random effects models were employed to estimate pooled effect sizes in order to reduce possible bias. Both Begg's and Egger's test were used to detect any potential publication bias within the meta-analyses (29,30). Significant publication bias was revealed if Begg's or Egger's $\mathrm{P}$ value $<0.05$.

We also conducted a sensitivity analysis, in which the impact of each study on the overall estimates could be detected by omitting individual studies sequentially, to further examine the stability of pooled estimates (27). The strong robustness of our meta-analysis was confirmed if there was no substantial variation between the adjusted results and the primary results (27). 
To evaluate the efficacy of ERAS programs on postoperative complications in detail, a meta-analysis was then performed on 4 subgroups: pulmonary, cardiovascular, urinary, and surgical complications. The relevant complications were judged according to the criteria used in recent large-registry trials based on the French Society of Thoracic and Cardiovascular Surgery (SFCTCV) database (31).

All statistical analyses were conducted using the Review Manager software (RevMan version 5.3, the Nordic Cochrane Center, the Cochrane Collaboration, 2014) and Stata (version 14; StataCorp LLC, College Station, TX, USA).

\section{Results}

\section{Literature search}

A flow diagram outlining the searching procedures is presented in Figure 2. A total of 1,204 potential articles were identified, including 648 PubMed citations, 398 EMBASE citations, and 153 Cochrane Library citations. In addition, a manual search of the reference lists also yielded 5 relevant studies. After checking for duplicates, reviewing titles, abstracts, and full texts, 22 eligible articles were included in qualitative assessment (32-53). A total of 21 articles were finally included in our meta-analysis after excluding a study due to it being of low quality (53).

\section{Characteristics of the included studies}

Baseline characteristics and major perioperative outcomes in each study are summarized in Tables 2 and 3. There were 19 cohort studies (34-52) and 2 RCTs $(32,33)$ included in this review. The publication dates of the included articles ranged from 2006 to 2020. The sample size of the trials ranged from 34 to 1,957 . All eligible articles were written in English.

\section{Patient characteristics}

Through layers of screening, 6,480 patients were finally enrolled in our meta-analysis. Detailed participant characteristics are displayed in Table 2 (more detailed baseline characteristics are presented in the Table S1). Approximately half of the participants were from North America ( $\mathrm{n}=3,332 ; 51.4 \%)$, followed by 1,827 patients from Europe (28.2\%), and 1321 patients from East Asia (20.4\%).
A total of 2,617 patients were included in the ERAS group $(40.4 \%)$, while 3863 patients were enrolled in the control group $(59.6 \%)$.

\section{ERAS elements}

Elaborate details of ERAS elements estimated in each study are summarized in Tables 4,5. The number of ERAS elements utilized in the ERAS group and control group ranged from 5 to 22 and 0 to 10 , respectively. The most commonly used ERAS element in our included studies was preadmission education/counselling (adopted by 19 studies), followed by early ambulation (adopted by 18 studies). The ERAS elements that were least used in eligible studies were alcohol dependency management and anemia management (adopted by 1 study). Although ERAS elements varied across these 21 studies, they overlapped for some common components, as shown in Tables 4,5.

\section{Quality assessment}

Quality assessment of the included studies is presented in Tables 6-8. Finally, all except for 1 of the included cohort studies (53) received a NOS score $\geq 6$. The mean NOS score of included studies was 6.84 [6-8], suggesting that they were of acceptable quality. As for RCTs $(32,33), 1$ of the 2 articles included was not double-blinded and did not mention the allocation concealment. Both articles did not mention whether the results included incomplete outcome data and selective reporting. There were no other risks of bias identified.

\section{Effect of ERAS on outcome}

\section{Postoperative complications}

As shown in Table 3, the overall postoperative complications rates in the ERAS group and the control group were $6.5-42.1 \%$ and $13.3-83.3 \%$, respectively. Of the 18 studies that reported on overall postoperative complications $(32-45,47,49-51)$, 9 reported that the ERAS group had decreased rates of overall postoperative complications $(34,36,37,39,40,42-45)$, while the other half reported no significant difference between the 2 groups $(32,33,35,38,41,47,49-51)$. The pooled RR of all 18 studies was 0.64 (95\% CI: 0.52 to $0.78 ; \mathrm{P}<0.0001$ ), as shown in Figure 3, indicating a significant decrease of overall complications rates in the ERAS group. However, a significant heterogeneity was found by a random effects 


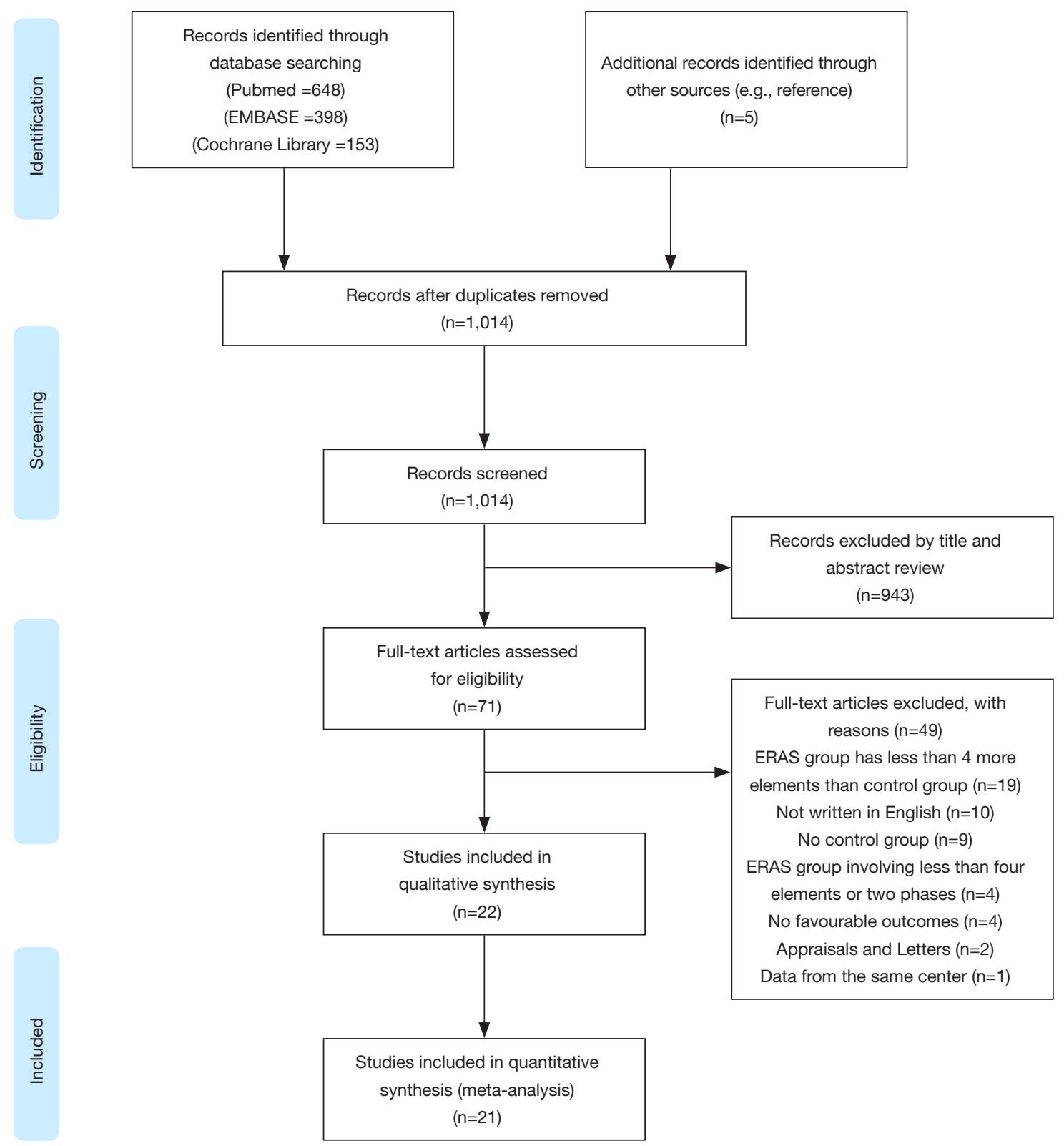

Figure 2 PRISMA flow diagram of literature retrieval. PRISMA, Preferred Reporting Items for Systematic Reviews and Meta-Analyses.

model ( $\mathrm{I}^{2}=63 \%$; $\left.\mathrm{P}<0.001\right)$. No publication bias was found using both Begg's test $(\mathrm{P}=0.363)$ and Egger's test $(\mathrm{P}=0.133)$.

To explore the source of high heterogeneity, we omitted the individual studies sequentially (27). Fortunately, we found a significantly reduced heterogeneity after excluding the studies performed by Gao et al. in $2015\left(\mathrm{I}^{2}=35 \%\right.$; $\mathrm{P}=0.08$ ), as shown in Figure 4 .

\section{In-hospital mortality}

A total of 13 studies reported in-hospital mortality
$(32,33,35,39-42,45-48,50,52)$, all of which reported no significant difference between the 2 groups. There were 11 deaths $(0.4 \%)$ in the ERAS group and 28 deaths $(0.7 \%)$ in the control group. However, 7 studies reported 0 events in both the experimental and control group (32,39$41,46,47,50)$; therefore, the pooled RD estimates with 95\% CIs were applied. The results showed no significant difference in the mortality rates between the ERAS group and the control group ( $\mathrm{RD}=0.00 ; 95 \% \mathrm{CI}$ : -0.01 to 0.00 ; $\mathrm{P}=0.62)$ with low heterogeneity $\left(\mathrm{I}^{2}=0 ; \mathrm{P}=1.00\right)$, as shown in 


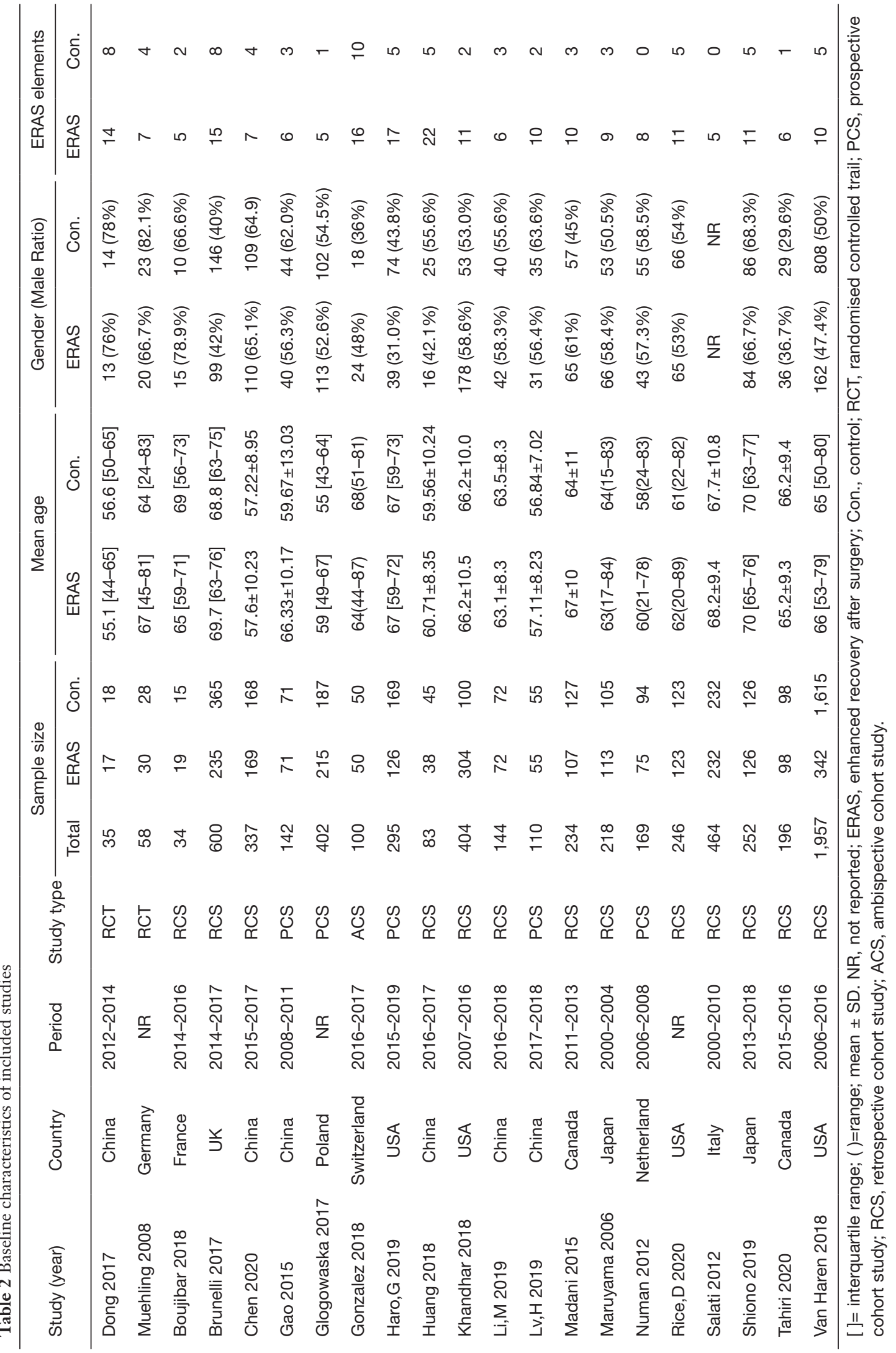




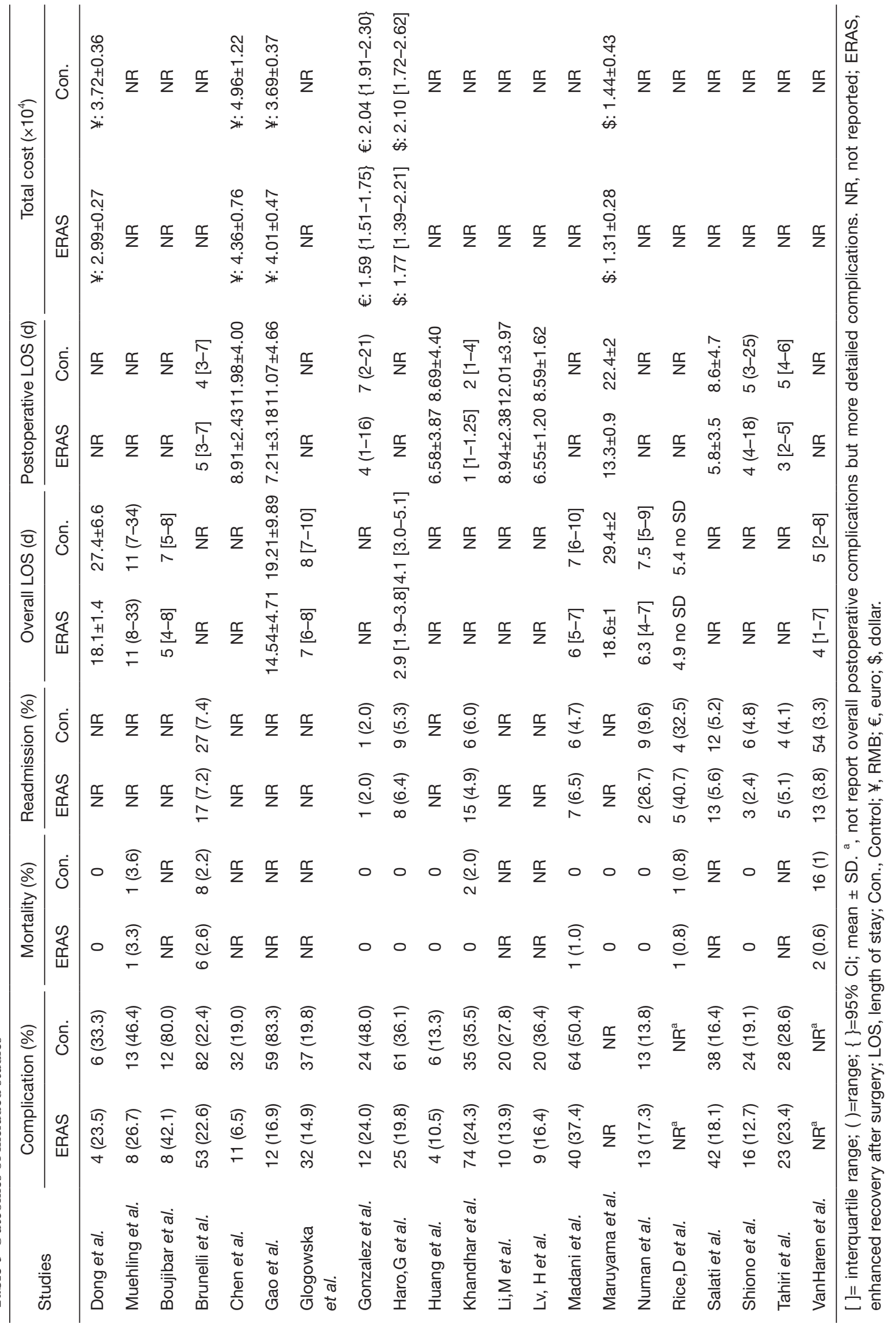


Table 4 Detailed ERAS elements of included studies (I)

\begin{tabular}{|c|c|c|c|c|c|c|c|c|c|c|c|}
\hline ERAS elements & \multicolumn{11}{|c|}{ Studies } \\
\hline Preadmission education/counselling & $\sqrt{ }$ & $\sqrt{ }$ & $\sqrt{ }^{\mathrm{a}}$ & $\sqrt{ }^{\mathrm{a}}$ & $\sqrt{ }$ & & $\sqrt{ }^{\mathrm{a}}$ & $\sqrt{ }^{\mathrm{a}}$ & $\sqrt{ }^{\mathrm{a}}$ & $\sqrt{ }^{\mathrm{a}}$ & $\sqrt{ }^{\mathrm{a}}$ \\
\hline Preoperative nutrition improvement & & & & $\sqrt{ }^{\mathrm{a}}$ & & & & $\sqrt{ }^{\mathrm{a}}$ & & $\sqrt{ }^{\mathrm{a}}$ & \\
\hline Alcohol dependency management & & & & & & & & & & $\sqrt{ }^{\mathrm{a}}$ & \\
\hline Anaemia management & & & & & & & & & & $\sqrt{ }^{\mathrm{a}}$ & \\
\hline $\begin{array}{l}\text { Preoperative cardiopulmonary function } \\
\text { assessment }\end{array}$ & $\sqrt{ }$ & & $\sqrt{ }$ & $\sqrt{ }$ & & $\sqrt{ }$ & $\sqrt{ }$ & & $\sqrt{ }^{\mathrm{a}}$ & $\sqrt{ }$ & $\sqrt{ }$ \\
\hline Venous thromboembolism prophylaxis & & & & & & & & $\sqrt{ }$ & $\sqrt{ }^{\mathrm{a}}$ & $\sqrt{ }^{\mathrm{a}}$ & \\
\hline Physical exercise training & & & $\sqrt{ }^{\mathrm{a}}$ & & $\sqrt{ }$ & $\sqrt{ }^{\mathrm{a}}$ & $\sqrt{ }^{a}$ & & & $\sqrt{ }^{\mathrm{a}}$ & $\sqrt{ }^{\mathrm{a}}$ \\
\hline \multicolumn{12}{|l|}{ Intraoperative interventions } \\
\hline $\begin{array}{l}\text { Prevention of intraoperative } \\
\text { hypothermia }\end{array}$ & $\sqrt{ }^{\mathrm{a}}$ & $\sqrt{ }$ & & $\sqrt{ }^{\mathrm{a}}$ & & & & $\sqrt{ }$ & & $\sqrt{ }^{\mathrm{a}}$ & $\sqrt{ }^{a}$ \\
\hline Standard anesthetic protocol & $\sqrt{ }$ & $\sqrt{ }$ & & $\sqrt{ }$ & & & & $\sqrt{ }$ & $\sqrt{ }$ & $\sqrt{ }$ & $\sqrt{ }^{\mathrm{a}}$ \\
\hline Protective lung ventilation & $\sqrt{ }$ & & & & & & & $\sqrt{ }$ & $\sqrt{ }^{a}$ & $\sqrt{ }$ & \\
\hline \multicolumn{12}{|l|}{ Postoperative interventions } \\
\hline $\begin{array}{l}\text { Postoperative airway management/ } \\
\text { pulmonary rehabilitation }\end{array}$ & & & & & $\sqrt{ }$ & & $\sqrt{ }^{a}$ & & $\sqrt{ }^{a}$ & $\sqrt{ }$ & \\
\hline Rational use of analgesics & $\sqrt{ }^{\mathrm{a}}$ & $\sqrt{ }^{\mathrm{a}}$ & & $\sqrt{ }^{\mathrm{a}}$ & $\sqrt{ }^{a}$ & $\sqrt{ }$ & & $\sqrt{ }$ & $\sqrt{ }$ & & $\sqrt{ }^{\mathrm{a}}$ \\
\hline PONV control & & & & $\sqrt{ }^{\mathrm{a}}$ & $\sqrt{ }^{\mathrm{a}}$ & & & $\sqrt{ }^{\mathrm{a}}$ & $\sqrt{ }^{\mathrm{a}}$ & $\sqrt{ }^{\mathrm{a}}$ & \\
\hline Standardized chest tube management & $\sqrt{ }$ & & & $\sqrt{ }$ & & & & $\sqrt{ }^{\mathrm{a}}$ & $\sqrt{ }^{a}$ & $\sqrt{ }^{\mathrm{a}}$ & \\
\hline Early removal of urinary catheter & $\sqrt{ }^{\mathrm{a}}$ & & & & $\sqrt{ }$ & & & & $\sqrt{{ }^{a}}$ & $\sqrt{ }{ }^{a}$ & \\
\hline Early oral feeding & $\sqrt{ }^{\mathrm{a}}$ & $\sqrt{ }^{\mathrm{a}}$ & & $\sqrt{ }^{\mathrm{a}}$ & & & & $\sqrt{ }$ & $\sqrt{ }^{\mathrm{a}}$ & & \\
\hline Early ambulation (out of bed) & $\sqrt{ }^{a}$ & $\sqrt{ }^{\mathrm{a}}$ & & $\sqrt{ }$ & $\sqrt{ }^{\mathrm{a}}$ & & $\sqrt{ }^{a}$ & $\sqrt{ }$ & $\sqrt{ }^{\mathrm{a}}$ & $\sqrt{ }^{\mathrm{a}}$ & $\sqrt{ }^{a}$ \\
\hline
\end{tabular}

${ }^{a}$, included in ERAS group but not in control group. ERAS, enhanced recovery after surgery; VATS, video-assisted thoracoscopic surgery; PONV, postoperative nausea and vomiting. 
Table 5 Detailed ERAS elements of included studies (II)

\begin{tabular}{|c|c|c|c|c|c|c|c|c|c|c|}
\hline ERAS elements & \multicolumn{10}{|c|}{ Studies } \\
\hline \multicolumn{11}{|l|}{ Preoperative interventions } \\
\hline Preadmission education/counselling & $\sqrt{ }$ & $\sqrt{ }$ & $\sqrt{ }^{\mathrm{a}}$ & $\sqrt{ }^{\mathrm{a}}$ & $\sqrt{ }^{\mathrm{a}}$ & $\sqrt{ }$ & $\sqrt{ }{ }^{a}$ & $\sqrt{ }^{a}$ & & $\sqrt{ }$ \\
\hline Preoperative nutrition improvement & & & & & & $\sqrt{ }^{\mathrm{a}}$ & & & & \\
\hline \multicolumn{11}{|l|}{ Alcohol dependency management } \\
\hline \multicolumn{11}{|l|}{ Anaemia management } \\
\hline $\begin{array}{l}\text { Preoperative Cardiopulmonary function } \\
\text { assessment }\end{array}$ & & & & & & & & $\sqrt{ }$ & & \\
\hline \multicolumn{11}{|l|}{ Venous thromboembolism prophylaxis } \\
\hline Physical exercise training & $\sqrt{ }$ & & $\sqrt{a}$ & & & & & $\sqrt{ }^{\mathrm{a}}$ & & \\
\hline \multicolumn{11}{|l|}{ Intraoperative interventions } \\
\hline Prevention of intraoperative hypothermia & & $\sqrt{ }^{a}$ & & & & & & & & \\
\hline Standard anesthetic protocol & & $\sqrt{a}$ & $\sqrt{ }$ & $\sqrt{ }$ & & $\sqrt{ }{ }^{a}$ & & $\sqrt{ }$ & & $\sqrt{ }{ }^{a}$ \\
\hline Protective lung ventilation & & & & $\sqrt{ }$ & & & & & & \\
\hline $\begin{array}{l}\text { Postoperative airway management/ } \\
\text { pulmonary rehabilitation }\end{array}$ & & $\sqrt{ }^{a}$ & $\sqrt{ }$ & & $\sqrt{a}$ & & $\sqrt{ }^{\mathrm{a}}$ & & $\sqrt{{ }^{a}}$ & \\
\hline Rational use of analgesics & $\sqrt{ }$ & $\sqrt{ }^{\mathrm{a}}$ & $\sqrt{ }$ & $\sqrt{a}$ & $\sqrt{ }^{\mathrm{a}}$ & $\sqrt{ }$ & & $\sqrt{ }$ & $\sqrt{ }^{a}$ & $\sqrt{ }^{\mathrm{a}}$ \\
\hline PONV control & & & & & & & & $\sqrt{ }$ & & \\
\hline Standardized chest tube management & $\sqrt{a}$ & $\sqrt{ }^{\mathrm{a}}$ & $\sqrt{ }{ }^{a}$ & $\sqrt{ }^{\mathrm{a}}$ & $\sqrt{ }{ }^{a}$ & $\sqrt{ }{ }^{\mathrm{a}}$ & $\sqrt{{ }^{a}}$ & $\sqrt{ }^{\mathrm{a}}$ & $\sqrt{ }^{\mathrm{a}}$ & $\sqrt{ }^{a}$ \\
\hline Early removal of urinary catheter & & $\sqrt{ }^{a}$ & $\sqrt{ }^{\mathrm{a}}$ & $\sqrt{ }^{a}$ & & & & & & \\
\hline Early oral feeding & & $\sqrt{ }{ }^{a}$ & $\sqrt{ }{ }^{\mathrm{a}}$ & $\sqrt{ }^{\mathrm{a}}$ & & $\sqrt{ }{ }^{a}$ & & $\sqrt{ }^{\mathrm{a}}$ & $\sqrt{a}$ & $\sqrt{ }^{\mathrm{a}}$ \\
\hline Early ambulation (out of bed) & $\sqrt{ }{ }^{a}$ & $\sqrt{ }{ }^{a}$ & $\sqrt{ }^{\mathrm{a}}$ & $\sqrt{ }{ }^{a}$ & $\sqrt{ }{ }^{a}$ & $\sqrt{ }$ & & $\sqrt{ }^{a}$ & $\sqrt{ }{ }^{a}$ & $\sqrt{ }$ \\
\hline
\end{tabular}

${ }^{a}$, included in ERAS group but not in control group. ERAS, enhanced recovery after surgery; VATS, video-assisted thoracoscopic surgery; PONV, postoperative nausea and vomiting. 
Table 6 Detailed quality assessment of cohort studies (I)

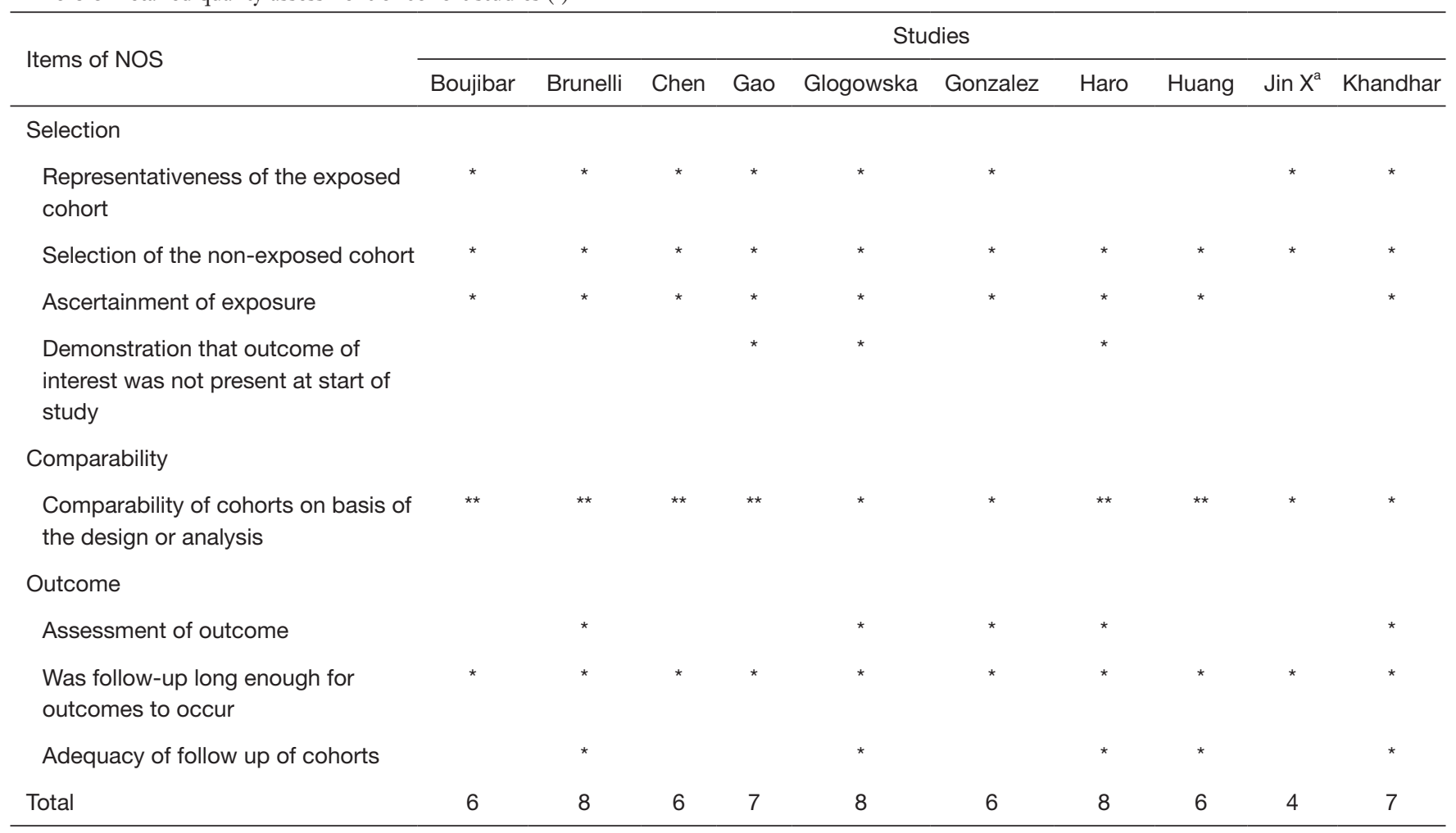

A study can be awarded a maximum of one star for each numbered item within the Selection and Outcome categories. A maximum of two stars can be given for Comparability. Studies rates $\geq 6$ are eligible. ${ }^{a}$, not qualified study. NOS, Newcastle-Ottawa Scale.

Figure 5. No publication bias was found using both Begg's test $(\mathrm{P}=1.00)$ and Egger's test $(\mathrm{P}=0.632)$.

\section{Readmission rate}

A total of 11 of the included studies mentioned the rates of readmission (35,39,40,42,45,47-52), and only 1 study found that the ERAS group had a lower readmission rate than the control group (47). There were 89 (4.9\%) and 138 (4.5\%) participants who were readmitted to hospital within 30 days in the ERAS group and control group, respectively. The pooled analysis of 11 available studies showed no significant difference in readmission rate between the ERAS group and control group ( $\mathrm{RR}=1.00$; $95 \% \mathrm{CI}$ : 0.76 to 1.32 ; $\mathrm{P}=0.99)$ with low heterogeneity $\left(\mathrm{I}^{2}=0 ; \mathrm{P}=0.90\right)$, as shown in Figure 6. No publication bias was found using both Begg's test $(\mathrm{P}=0.276)$ and Egger's test $(\mathrm{P}=0.305)$.

\section{LOS}

Regarding LOS, we analyzed 2 outcomes: overall LOS and postoperative LOS. A total of 11 studies reported overall LOS (32-34,37,38,40,45-48,52) and 12 studies reported postoperative LOS (35-37,39,41-44,46,49-51). However, only 3 studies presented overall LOS in the format of mean $\pm \mathrm{SD}(32,37,46)$; therefore, we performed a qualitative analysis instead of meta-analysis of overall LOS. Among the 11 studies that reported overall LOS, 9 indicated that the ERAS-treated patients had a significantly shortened overall LOS $(32,37,38,40,45-48,52)$, while the remaining 2 studies found that the difference was not significant $(33,34)$, as shown in Table 3. Among the 12 studies reporting postoperative LOS, all but 1 (35) reported significantly shortened postoperative LOS in the ERAS group. However, only 7 provided the data of mean \pm SD $(36,37,41,43,44,46,49)$. The pooled analysis of 7 eligible studies indicated that participants in the ERAS group had significantly shorter postoperative LOS (SMD $=-1.58$; 95\% CI: -2.38 to $-0.79 ; \mathrm{P}<0.0001)$ with a significantly high heterogeneity $\left(\mathrm{I}^{2}=98 \% ; \mathrm{P}<0.0001\right)$ between studies (Figure 7). No publication bias was found using both Begg's test $(\mathrm{P}=0.072)$ and Egger's test $(\mathrm{P}=0.113)$. 
Table 7 Detailed quality assessment of cohort studies (II)

\begin{tabular}{|c|c|c|c|c|c|c|c|c|c|c|}
\hline Items of NOS & \multicolumn{10}{|c|}{ Studies } \\
\hline \multicolumn{11}{|l|}{ Selection } \\
\hline $\begin{array}{l}\text { Representativeness of the exposed } \\
\text { cohort }\end{array}$ & * & * & * & * & * & * & * & * & * & * \\
\hline $\begin{array}{l}\text { Demonstration that outcome of interest } \\
\text { was not present at start of study }\end{array}$ & & * & & & * & & & & & \\
\hline \multicolumn{11}{|l|}{ Comparability } \\
\hline $\begin{array}{l}\text { Comparability of cohorts on basis of } \\
\text { the design or analysis }\end{array}$ & $\star *$ & $\star *$ & ** & $\star \star$ & ** & ** & ** & ** & * & * \\
\hline Assessment of outcome & & & * & & * & * & * & * & * & * \\
\hline $\begin{array}{l}\text { Was follow-up long enough for } \\
\text { outcomes to occur }\end{array}$ & * & * & * & * & & * & * & * & * & * \\
\hline Adequacy of follow up of cohorts & & & & & * & * & & & & \\
\hline Total & 6 & 7 & 7 & 6 & 8 & 8 & 7 & 7 & 6 & 6 \\
\hline
\end{tabular}

A study can be awarded a maximum of one star for each numbered item within the Selection and Outcome categories. A maximum of two stars can be given for Comparability. Studies rates $\geq 6$ are eligible. ${ }^{a}$, not qualified study. NOS, Newcastle-Ottawa Scale.

Table 8 Detailed quality assessment of RCTs

\begin{tabular}{lcc}
\hline & & \multicolumn{2}{c}{ Studies } \\
\hline Items of Cochrane Collaboration's Quality assessment Tool & Dong et al. & Muehling et al. \\
\cline { 2 - 3 } Random sequence generation (selection bias) & $(+)$ & $(+)$ \\
Allocation concealment (selection bias) & $(+)$ & $($ ?) \\
Blinding of participants and personnel (performance bias) & $(+)$ & $(-)$ \\
Blinding of outcome assessment (detection bias) & $(+)$ & $(-)$ \\
Incomplete outcome data (attrition bias) & $(?)$ & $(?)$ \\
Selective reporting (reporting bias) & $(+)$ & $(?)$ \\
Other bias & 5 & $(+)$ \\
Total & & 2 \\
\hline
\end{tabular}

(+): low risk of bias; (-): high risk of bias; (?): unclear risk of bias. RCTs, randomised controlled trails.

\section{Total cost of hospitalization}

A total of 6 studies mentioned total cost of hospitalization $(32,36,37,39,40,46)$, but only 4 studies presented data as mean \pm SD $(32,36,37,46)$. Due to the small number of studies reporting this outcome, we performed a qualitative review instead of a meta-analysis. Of these 4 studies, 3 reported a significantly lower cost of hospitalization in the ERAS group $(32,36,46)$; however, the studies performed by Gao et al. in 2015 found that the ERAS group had a much higher cost of hospitalization (37). The remaining 2 studies 


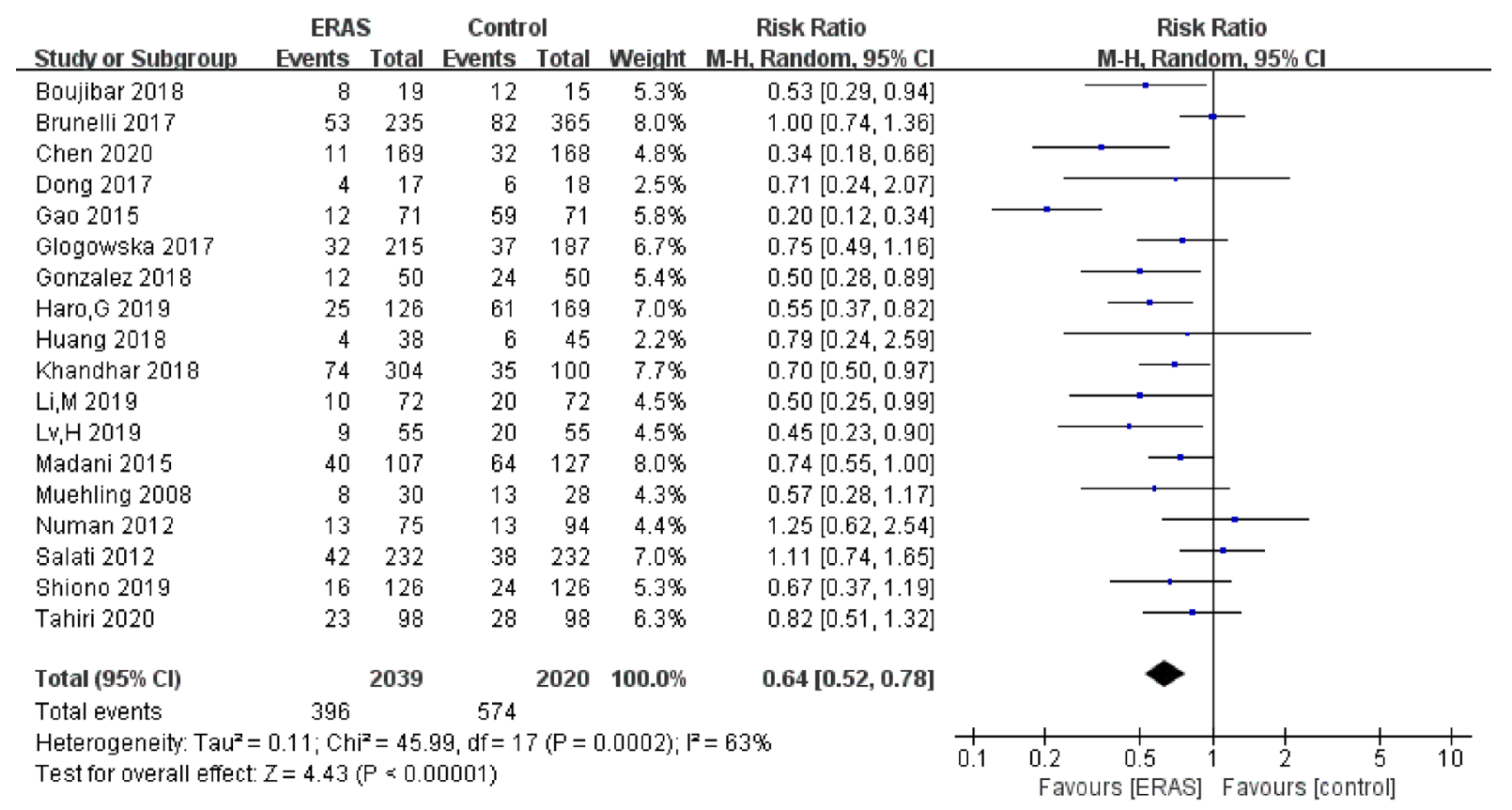

Figure 3 Meta-analysis of overall postoperative complications in the ERAS vs. control group. ERAS, enhanced recovery after surgery.

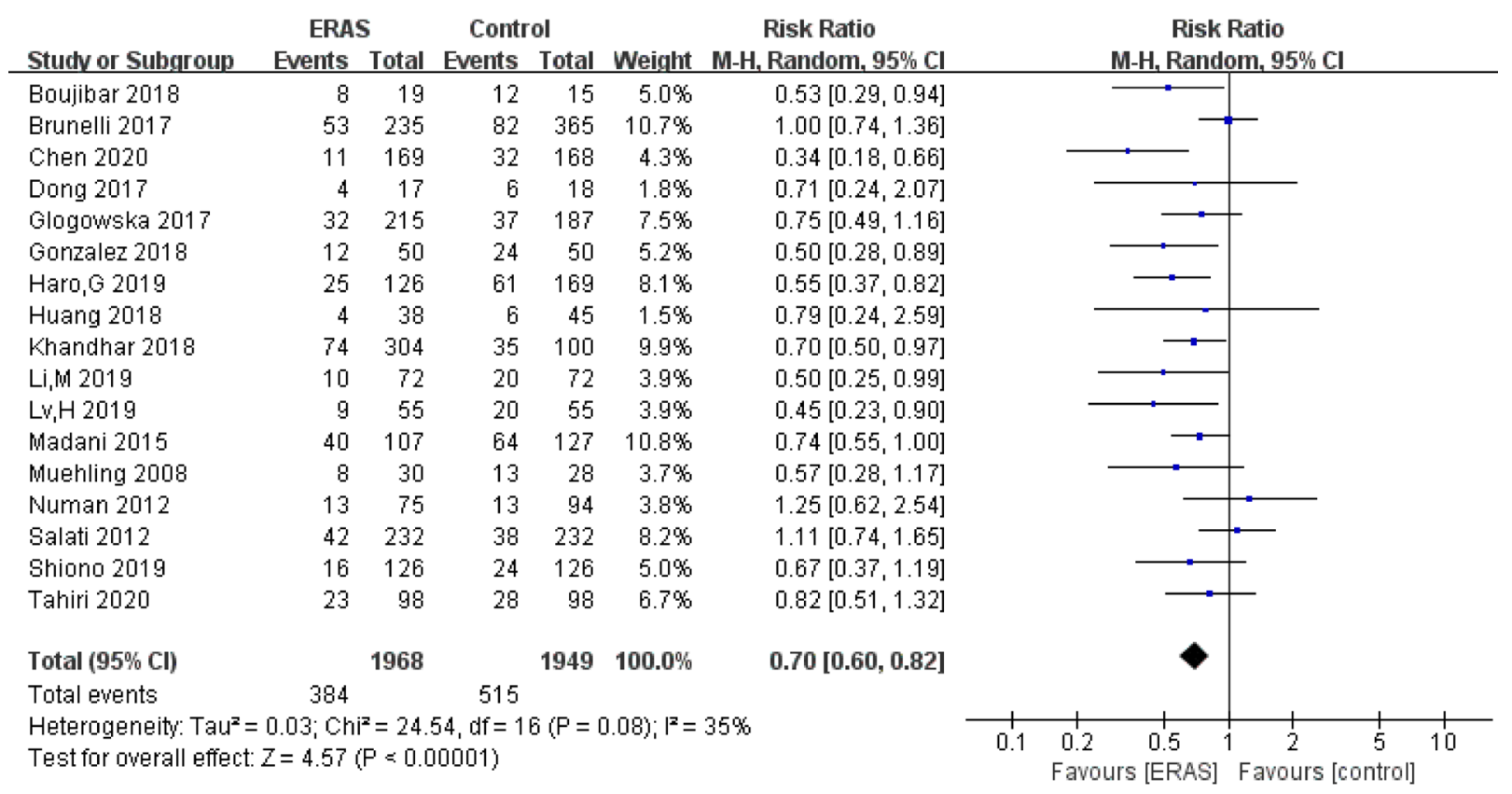

Figure 4 Meta-analysis of overall postoperative complications in ERAS vs. control group after excluding the study by Gao et al. ERAS, enhanced recovery after surgery. 


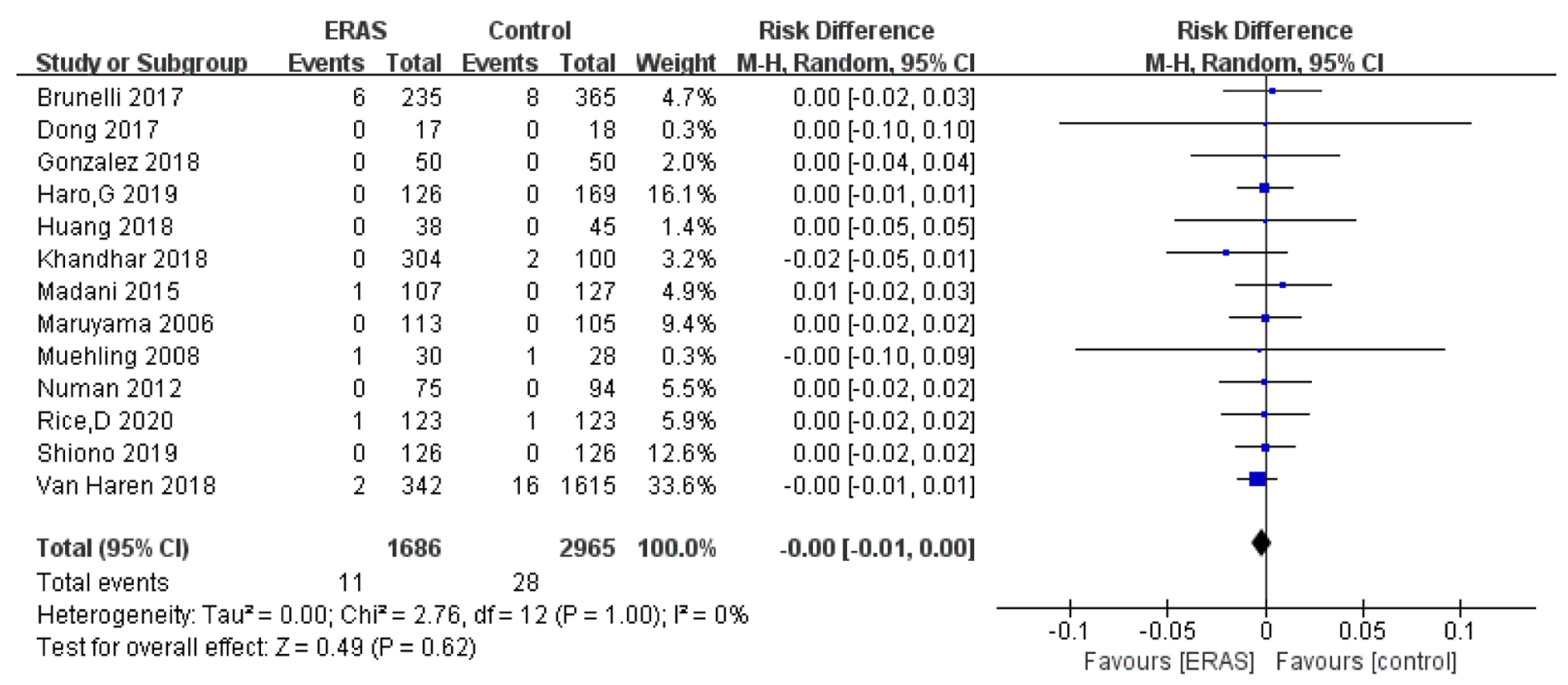

Figure 5 Meta-analysis of in-hospital mortality in the ERAS $v s$. control group. ERAS, enhanced recovery after surgery.

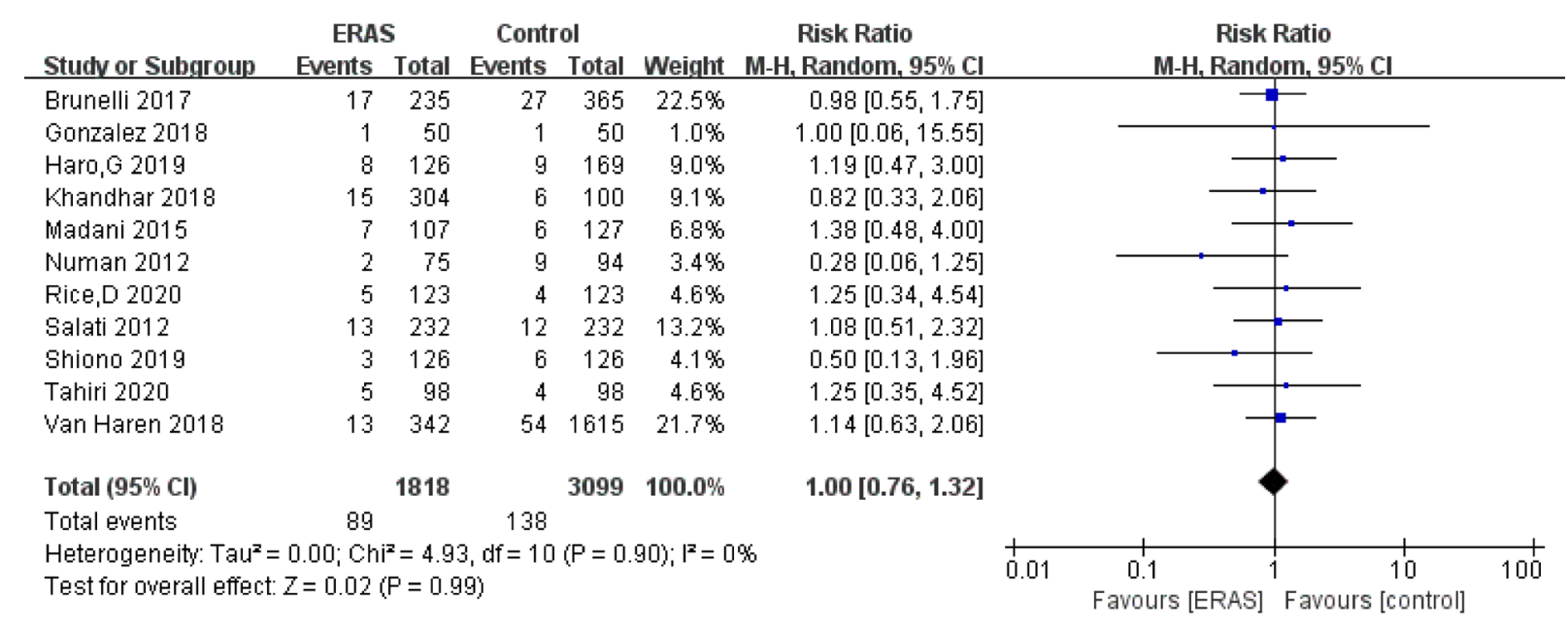

Figure 6 Meta-analysis of readmission rate in the ERAS vs. control group. ERAS, enhanced recovery after surgery.

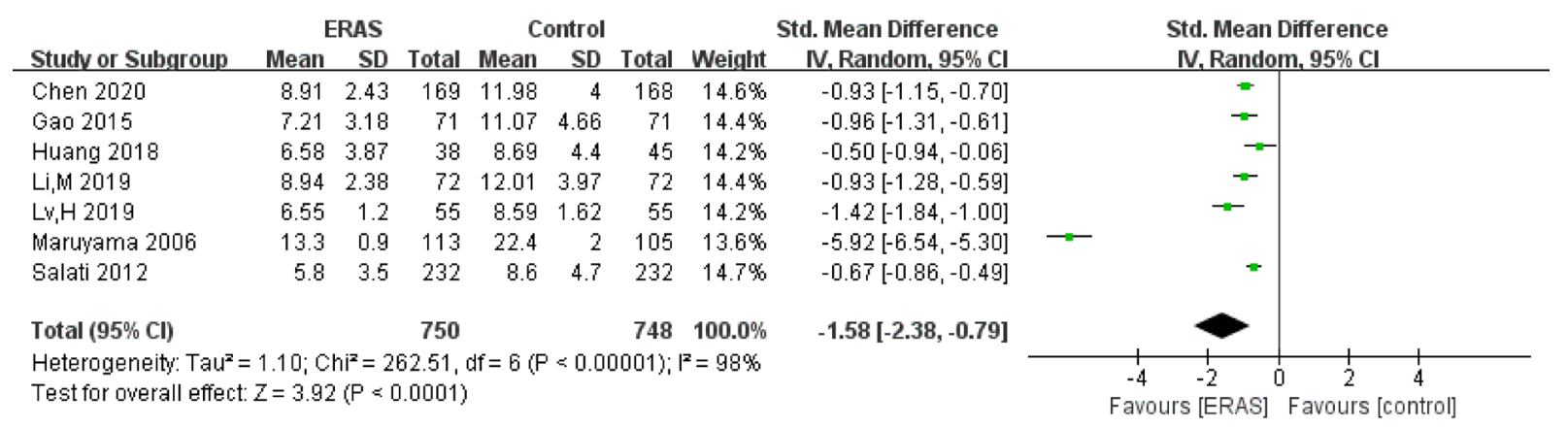

Figure 7 Meta-analysis of postoperative LOS in the ERAS vs. control group. LOS, length of stay; ERAS, enhanced recovery after surgery. 
Table 9 Meta-analysis for effects of the ERAS program on postoperative complications

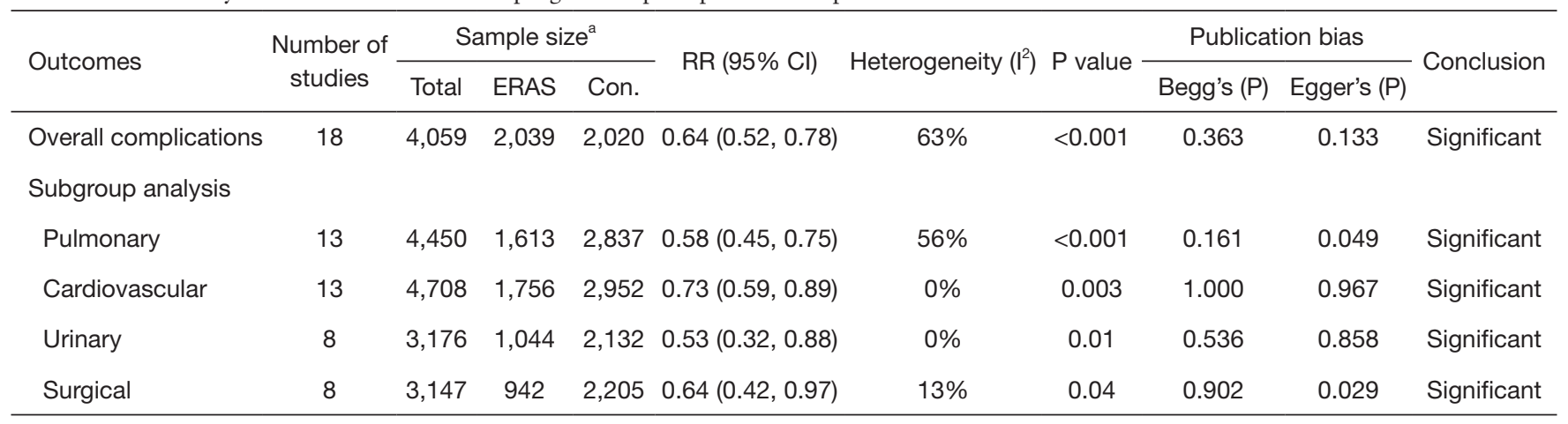

a, studies of Van Haren and Rice D did not provide overall postoperative complications but detailed data of subgroup complications. ERAS, enhanced recovery after surgery; RR, relative risk; Con., control; Cl, confidence interval.

reported data as a median with interquartile range (IQR) or $95 \%$ CI $(39,40)$, both of which showed a lower total cost in the ERAS group than in the control group, as shown in Table 3.

\section{Subgroup analysis}

In order to assess the effects of ERAS programs on postoperative complications in detail, we performed a subgroup analysis as follows: pulmonary complications, cardiovascular complications, urinary complications, and surgical complications. There were 13 studies included in the subgroup of pulmonary complications $(32,33,35$ $37,39,41-45,48,52), 13$ in the subgroup of cardiovascular complications $(32,33,35-39,41,42,44,45,48,52)$, 8 in the subgroup of urinary complications $(32,33,37,39,42,45,48,52)$, and 8 in the subgroup of surgical complications (33,37-39,43-45,52).

The meta-analysis of results in all subgroups are summarized in Table 9. As shown in Figure 8, the synthesized estimates using random effects model revealed that all subgroups of complications were significantly decreased in the patients who were treated with the ERAS program. However, the meta-analysis of the pulmonary subgroup showed a moderate heterogeneity $\left(\mathrm{I}^{2}=55.6 \% ; \mathrm{P}=0.008\right)$. The other 3 subgroup analyses did not present any significant heterogeneity. Egger's test detected a slight publication bias of meta-analysis of pulmonary complications subgroup $(\mathrm{P}=0.049)$ and surgical complication subgroup $(\mathrm{P}=0.029)$. No other publication bias was found using both Begg's test and Egger's test in other subgroup analyses, as shown in Table 9.

\section{Sensitivity analysis}

We performed sensitivity analysis by omitting individual studies sequentially. None of the pooled RRs based on the remaining studies in each group of meta-analysis was out of the estimated range, as shown in Figure 9. No substantial variation was found between adjusted pooled estimates and primary pooled estimates. The strong robustness of our meta-analysis was thus confirmed.

\section{Discussion}

Through this meta-analysis, we found that patients treated with the ERAS program had a lower risk of developing postoperative complications and a decreased postoperative LOS. However, in terms of in-hospital mortality and readmission rate, there was no significant difference between the ERAS group and the control group. Qualitative assessment revealed significantly decreased overall LOS and total hospitalization costs in the ERAS group.

The role of the ERAS program in lung cancer surgery has been extensively studied, and the ERAS Association has formulated detailed guidelines, illustrating the benefits of its various components $(24,25)$. According to our best knowledge, this review is an updated meta-analysis to explore the impact of the ERAS program on patients undergoing lung cancer surgery. This meta-analysis covers all the provisions of the standardized PRISMA report (23). Compared with previous studies $(20,21)$, ours has the following advantages. To begin with, in order to increase the sample size and improve the credibility of the results, we included both RCTs and cohort studies, 


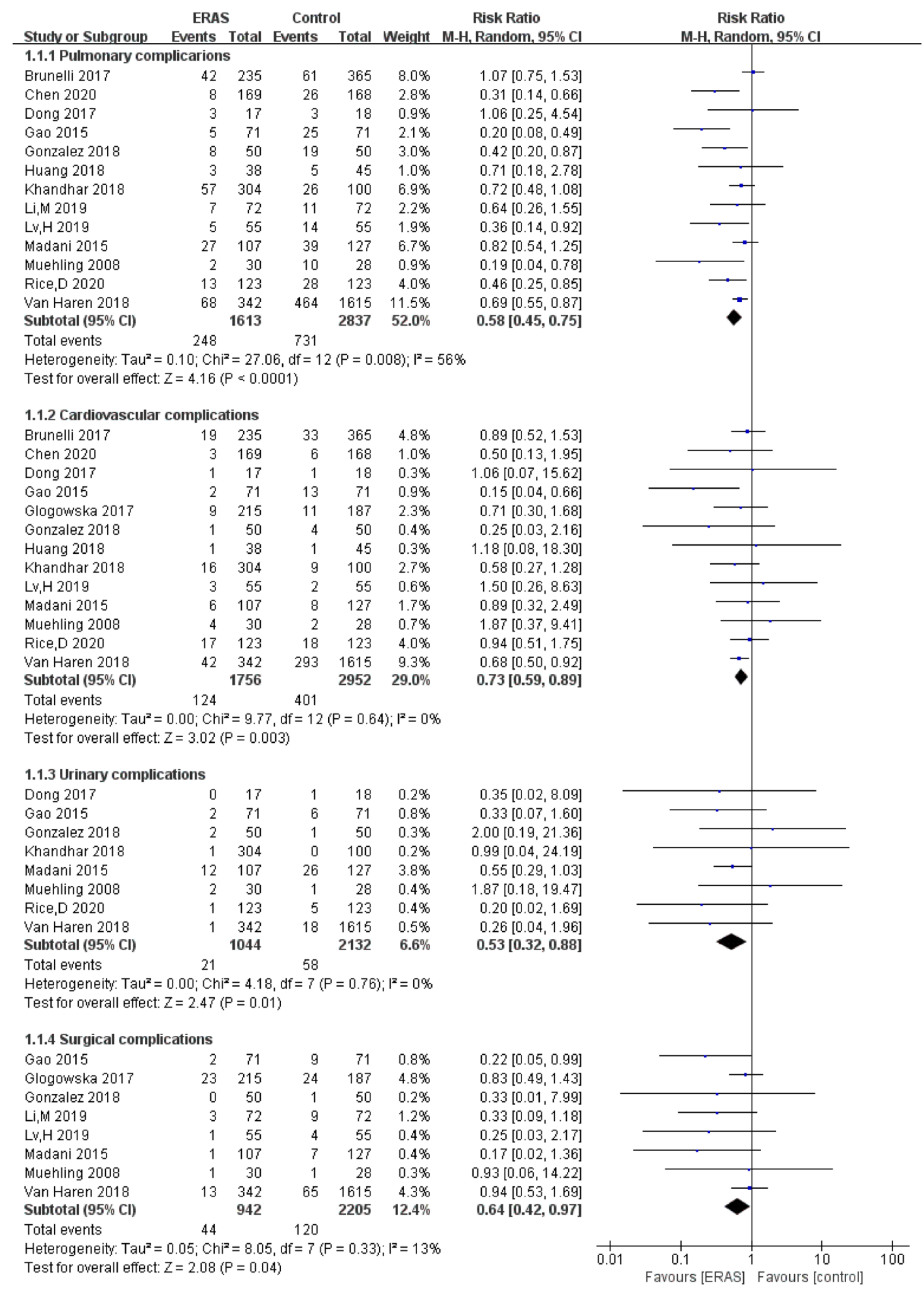

Figure 8 Subgroup analyses of the effects of the ERAS program on pulmonary, cardiovascular, urinary, and surgical complications following lung cancer surgery. ERAS, enhanced recovery after surgery. 


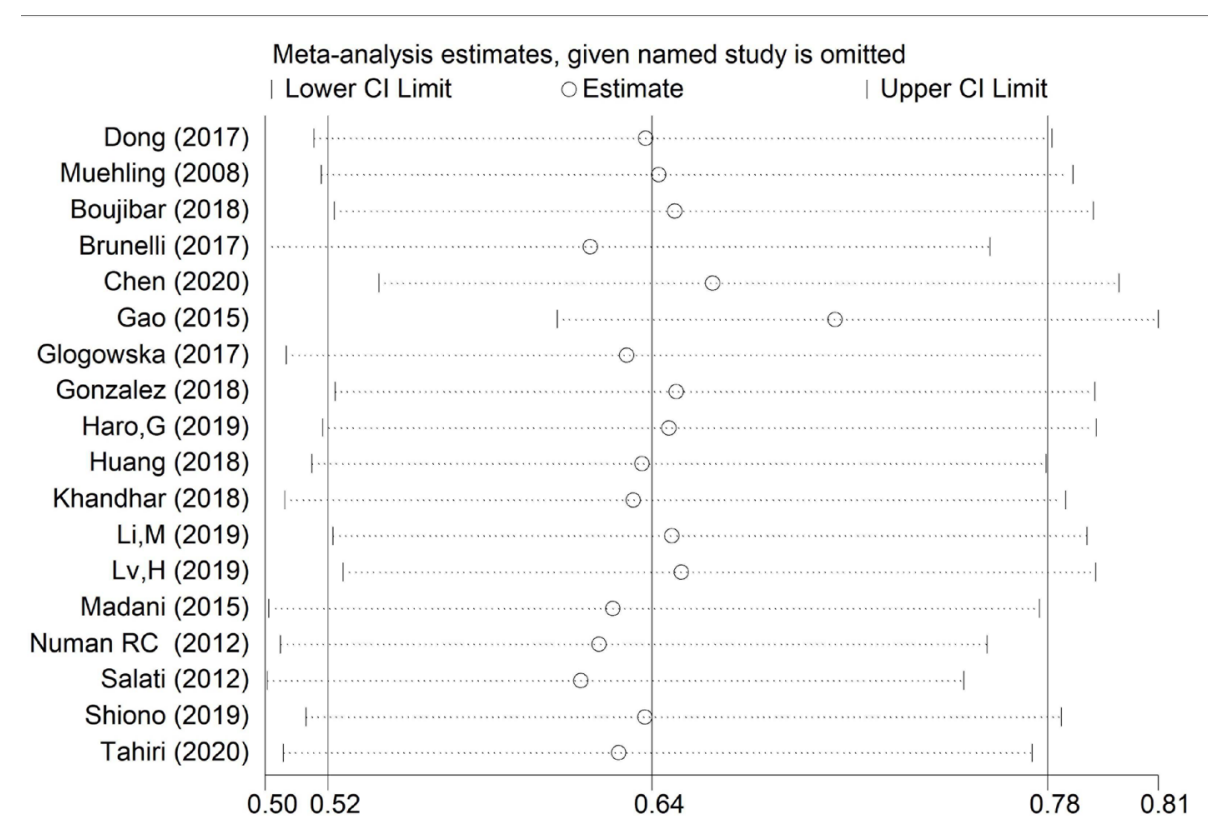

Figure 9 Sensitivity analysis of meta-analysis.

involving 10 countries and regions. Second, according to the recently published guidelines and previous review (19-21,24,25), we formulated a fresh ERAS protocol composed of 25 elements covering 3 phases. Meanwhile, we emphasized the difference between the ERAS group and the control group (the difference between the 2 groups needed exceed 3 ERAS elements), which was different from Li's study (21). Third, we analyzed the impact of the ERAS program on readmission rate and postoperative LOS and conducted a more comprehensive subgroup analysis including pulmonary, cardiovascular, urinary, and surgical complications. Therefore, this review has become the latest and most comprehensive review of the ERAS literature on lung cancer surgery.

Lung surgery can precipitate a series of physical and psychological stress responses, which can cause critical trauma to the body and postoperative complications $(54,55)$. The ERAS program can effectively reduce the psychological and physical stress of patients, thereby reducing the occurrence of postoperative complications $(56,57)$. Postoperative inflammatory response is the main cause of postoperative complications $(58,59)$. The study by Dong et al. (32) showed that postoperative inflammatory factors such as interleukin-6 (IL-6) in the ERAS group were significantly reduced, indicating that the ERAS program can weaken the postoperative inflammatory response of patients. Notably, we found that ERAS can effectively reduce the incidence of postoperative cardiovascular complications, which is in contrast to the results of previous studies $(20,21)$.

The meta-analysis of overall postoperative complications showed a high heterogeneity $\left(\mathrm{I}^{2}=63 \% ; \mathrm{P}<0.001\right)$. Fortunately, after excluding Gao's study from 2015 (37), the heterogeneity of the meta-analysis was reduced significantly $\left(\mathrm{I}^{2}=35 \% ; \mathrm{P}=0.08\right)$. Hence, we focused on Gao's study. In this study, the author included 142 high-risk patients (heavy smokers, airway hyperresponsiveness, hypopulmonary function) as the participants. The complication rate of the control group was $83.31 \%, 5$ times more than that of the ERAS group (16.9\%), which far exceeded the results of other studies. The probability of postoperative complications is much higher for high-risk patients than for those with normal risk due to deficient lung function, more microbial colonization in the respiratory tract, and low immunity $(60,61)$. Meanwhile, some components in the ERAS program, such as preoperative assessment of cardiopulmonary function, preoperative exercise, prophylactic antibiotics, and the application of respiratory drugs could significantly improve the lung function of high-risk patients and enhance their tolerance to surgery (24), thereby reducing the incidence of postoperative complications to a greater extent. Muehling et al. performed a subgroup analysis of patients with FEV $1 \%<75 \%$ in 2008 , and found the same results (33). 
This further implies that the ERAS program may bring greater benefits to high-risk patients, which can become the focus of future research.

Compared with the previous meta-analysis, one novelty of our research was the analysis of the impact of the ERAS program on the readmission rate. The results indicated that the readmission rate of patients in the ERAS group did not increase significantly compared with the control group. However, some of the eligible studies were not sufficiently transparent in terms of readmission, which might have reduced the credibility of the results to some extent. In addition, there was a significant reduction in LOS and no significant increase in in-hospital mortality, which demonstrated that the implementation of the ERAS program in lung cancer surgery can effectively accelerate postoperative recovery without threating patient safety.

Although this review found that the patients treated with the ERAS program have a significantly shortened postoperative LOS, it also showed significantly high heterogeneity $\left(\mathrm{I}^{2}=98 \% ; \mathrm{P}<0.001\right)$. The reasons for this heterogeneity may be various and include variability in the ERAS components, surgical procedures, inclusion criteria, and traditional control standards between countries. Heterogeneity can also be attributed to some clinical factors, such as the technical level of hospitals and surgeons, patient compliance with the ERAS program, surgical procedures, conflicting evaluations of results, and others.

The cost-benefit analysis conducted in our study also indicated that the ERAS program has obvious economic benefits for lung cancer surgery. Among the 6 studies mentioning hospitalization expenses, 5 articles (based on 1,127 patients) reported that the hospitalization expenses of the ERAS group were significantly lower than those of the control group. In contrast, the study conducted by Gao et al. in 2015 showed that the overall hospitalization costs of patients in the ERAS group were slightly higher than those in the control group, which might have been due to the prolonged preoperative hospital stay and the additional cost of pulmonary rehabilitation (approximately 1,000 RMB) (37). It might also have been caused by differences in economic and medical expenses between countries. Unfortunately, we are unable to further assess the impact of the huge differences in the socioeconomic level and health care between the East and the West.

In other surgical fields, minimally invasive surgery serves as a key element of the ERAS program (62). It has been shown that VATS reduces complications after lung resection and effectively shortens the length of hospital stay $(6,63)$.
Conceptually, VATS seems to be the ideal surgical method for the ERAS program to improve postoperative results. Therefore, we originally planned to conduct a subgroup analysis on the impact of ERAS on open surgery and VATS, but due to the insufficient data on open surgery, our idea was not implemented, but could be considered in a future meta-analysis.

This review had some limitations. First, the majority of the included studies were cohort studies, and only 2 RCTs were included. Moreover, the majority of eligible cohort studies were separate-sample pre-post-test designs. These types of studies have some limitations, such as nonparallel controls and cohort selection, which might have introduced biases and reduced the reliability of the results. Second, the ERAS protocols of the included studies were significantly different, and the implementation standards of the ERAS program varied between each country and region, possibly producing bias and reducing the credibility of the results. In addition, there were differences in patient compliance with the ERAS program $(64,65)$, which might have led to obvious heterogeneity in the results. Third, we did not analyze patient-reported outcomes such as pain score and QoL because published research did not provide these types of data. We encourage future research to be more focused on this aspect. Finally, a certain language-based bias might have arisen due to the requirement of full-text English language literature; future meta-analyses should include studies written in other languages.

\section{Conclusions}

This meta-analysis is the most up-to-date and comprehensive review of the literature on the ERAS program for lung cancer surgery. Implementation of the ERAS program in lung cancer surgery can not only significantly reduce the risks of overall postoperative complications and hospitalization costs, but also shorten the LOS without increasing the rate of in-hospital mortality and readmission. In short, the ERAS program is an effective and safe strategy and should be recommended for lung cancer surgery.

\section{Acknowledgments}

We thank AME Editing Service (http://editing.amegroups. cn/\#editing) for its linguistic assistance during the preparation of this manuscript.

Funding: This work was supported by the National Natural 
Science Foundation of China (no. 8162292, 81802397), the Jinan Science and Technology Bureau (no. 2019GXRC051), the Natural Science Foundation of Shandong Province (no. ZR2017BH035), and the Taishan Scholar Program of Shandong Province (no. ts201712087).

\section{Footnote}

Reporting Checklist: The authors have completed the PRISMA reporting checklist. Available at https://dx.doi. org/10.21037/jtd-21-433

Peer Review File: Available at https://dx.doi.org/10.21037/ jtd-21-433

Conflicts of Interest: All authors have completed the ICMJE uniform disclosure form (available at https://dx.doi. org/10.21037/jtd-21-433). The authors have no conflicts of interest to declare.

Ethical Statement: The authors are accountable for all aspects of the work in ensuring that questions related to the accuracy and integrity of any part of the work are appropriately investigated and resolved.

Open Access Statement: This is an Open Access article distributed in accordance with the Creative Commons Attribution-NonCommercial-NoDerivs 4.0 International License (CC BY-NC-ND 4.0), which permits the noncommercial replication and distribution of the article with the strict proviso that no changes or edits are made and the original work is properly cited (including links to both the formal publication through the relevant DOI and the license). See: https://creativecommons.org/licenses/by-nc-nd/4.0/.

\section{References}

1. Nasim F, Sabath BF, Eapen GA. Lung Cancer. Med Clin North Am 2019;103:463-73.

2. Mao Y, Yang D, He J, et al. Epidemiology of Lung Cancer. Surg Oncol Clin N Am 2016;25:439-45.

3. Siegel RL, Miller KD, Jemal A. Cancer statistics, 2020. CA Cancer J Clin 2020;70:7-30.

4. Chen $\mathrm{W}$, Zheng R, Baade PD, et al. Cancer statistics in China, 2015. CA Cancer J Clin 2016;66:115-32.

5. Flores RM, Alam N. Video-assisted thoracic surgery lobectomy (VATS), open thoracotomy, and the robot for lung cancer. Ann Thorac Surg 2008;85:S710-5.
6. Falcoz PE, Puyraveau M, Thomas PA, et al. Videoassisted thoracoscopic surgery versus open lobectomy for primary non-small-cell lung cancer: a propensitymatched analysis of outcome from the European Society of Thoracic Surgeon database. Eur J Cardiothorac Surg 2016;49:602-9.

7. Seely AJ, Ivanovic J, Threader J, et al. Systematic classification of morbidity and mortality after thoracic surgery. Ann Thorac Surg 2010;90:936-42; discussion 42.

8. Choi SM, Lee J, Park YS, et al. Postoperative pulmonary complications after surgery in patients with interstitial lung disease. Respiration 2014;87:287-93.

9. Phillips JD, Merkow RP, Sherman KL, et al. Factors affecting selection of operative approach and subsequent short-term outcomes after anatomic resection for lung cancer. J Am Coll Surg 2012;215:206-15.

10. Kehlet H, Wilmore DW. Evidence-based surgical care and the evolution of fast-track surgery. Ann Surg 2008;248:189-98.

11. Ljungqvist O, Scott M, Fearon KC. Enhanced Recovery After Surgery: A Review. JAMA Surg 2017;152:292-8.

12. Engelman RM, Rousou JA, Flack JE, et al. Fast-track recovery of the coronary bypass patient. Ann Thorac Surg 1994;58:1742-6.

13. Ni X, Jia D, Chen Y, et al. Is the Enhanced Recovery After Surgery (ERAS) Program Effective and Safe in Laparoscopic Colorectal Cancer Surgery? A Meta-Analysis of Randomized Controlled Trials. J Gastrointest Surg 2019;23:1502-12.

14. Damania R, Cocieru A. Impact of enhanced recovery after surgery protocols on postoperative morbidity and mortality in patients undergoing routine hepatectomy: review of the current evidence. Ann Transl Med 2017;5:341.

15. Keil DS, Schiff LD, Carey ET, et al. Predictors of Admission After the Implementation of an Enhanced Recovery After Surgery Pathway for Minimally Invasive Gynecologic Surgery. Anesth Analg 2019;129:776-83.

16. Zhao Y, Zhang S, Liu B, et al. Clinical efficacy of enhanced recovery after surgery (ERAS) program in patients undergoing radical prostatectomy: a systematic review and meta-analysis. World J Surg Oncol 2020;18:131.

17. Elsarrag M, Soldozy S, Patel P, et al. Enhanced recovery after spine surgery: a systematic review. Neurosurg Focus 2019;46:E3.

18. Swaminathan N, Kundra P, Ravi R, et al. ERAS protocol with respiratory prehabilitation versus conventional perioperative protocol in elective gastrectomy- a randomized controlled trial. Int J Surg 2020;81:149-57. 
19. Nicholson A, Lowe MC, Parker J, et al. Systematic review and meta-analysis of enhanced recovery programmes in surgical patients. Br J Surg 2014;101:172-88.

20. Fiore JF, Bejjani J, Conrad K, et al. Systematic review of the influence of enhanced recovery pathways in elective lung resection. J Thorac Cardiovasc Surg 2016;151:708-17.e6.

21. Li S, Zhou K, Che G, et al. Enhanced recovery programs in lung cancer surgery: Systematic review and metaanalysis of randomized controlled trials. Cancer Manag Res 2017;9:657-70.

22. Stroup DF, Berlin JA, Morton SC, et al. Meta-analysis of Observational Studies in Epidemiology. JAMA 2000;283:2008-12.

23. Liberati A, Altman DG, Tetzlaff J, et al. The PRISMA statement for reporting systematic reviews and metaanalyses of studies that evaluate healthcare interventions: explanation and elaboration. BMJ 2009;339:b2700.

24. Batchelor TJP, Rasburn NJ, Abdelnour-Berchtold E, et al. Guidelines for enhanced recovery after lung surgery: recommendations of the Enhanced Recovery After Surgery (ERAS(R)) Society and the European Society of Thoracic Surgeons (ESTS). Eur J Cardiothorac Surg 2019;5 5:91-115.

25. Gao S, Barello S, Chen L, et al. Clinical guidelines on perioperative management strategies for enhanced recovery after lung surgery. Transl Lung Cancer Res 2019;8:1174-87.

26. Stang A. Critical evaluation of the Newcastle-Ottawa scale for the assessment of the quality of nonrandomized studies in meta-analyses. Eur J Epidemiol 2010;25:603-5.

27. Higgins JPT, Thomas J, Chandler J, Cumpston M, Li T, Page MJ, Welch VA (editors). Cochrane Handbook for Systematic Reviews of Interventions version 6.1 (updated September 2020). Cochrane, 2020. Available online: www. training.cochrane.org/handbook.

28. Higgins JP, Thompson SG. Quantifying heterogeneity in a meta-analysis. Stat Med 2002;21:1539-58.

29. Begg CB, Mazumdar M. Operating Characteristics of a Rank Correlation Test for Publication Bias. BIOMETRICS 1994;50:1088-101.

30. Egger M, Smith GD, Schneider M, et al. Bias in metaanalysis detected by a simple, graphical test. BMJ 1997;315:629-34.

31. Thomas PA, Berbis J, Baste JM, et al. Pneumonectomy for lung cancer: contemporary national early morbidity and mortality outcomes. J Thorac Cardiovasc Surg 2015;149:73-82.

32. Dong Q, Zhang K, Cao S, et al. Fast-track surgery versus conventional perioperative management of lung cancerassociated pneumonectomy: A randomized controlled clinical trial. World J Surg Oncol 2017;15:20.

33. Muehling BM, Halter GL, Schelzig H, et al. Reduction of postoperative pulmonary complications after lung surgery using a fast track clinical pathway. Eur J Cardiothorac Surg 2008;34:174-80.

34. Boujibar F, Bonnevie T, Debeaumont D, et al. Impact of prehabilitation on morbidity and mortality after pulmonary lobectomy by minimally invasive surgery: a cohort study. J Thorac Dis 2018;10:2240-8.

35. Brunelli A, Thomas C, Dinesh P, et al. Enhanced recovery pathway versus standard care in patients undergoing videoassisted thoracoscopic lobectomy. J Thorac Cardiovasc Surg 2017;154:2084-90.

36. Chen F, Wang G. Enhanced Recovery after Surgery for Lung Cancer Patients. Open Med (Wars) 2020;15:198-203.

37. Gao K, Yu PM, Su JH, et al. Cardiopulmonary exercise testing screening and pre-operative pulmonary rehabilitation reduce postoperative complications and improve fast-track recovery after lung cancer surgery: A study for 342 cases. Thoracic Cancer 2015;6:443-9.

38. Glogowska O, Glogowski M, Szmit S. Intensive rehabilitation as an independent determinant of better outcome in patients with lung tumors treated by thoracic surgery. Arch Med Sci 2017;13:1442-8.

39. Gonzalez M, Abdelnour-Berchtold E, Perentes JY, et al. An enhanced recovery after surgery program for videoassisted thoracoscopic surgery anatomical lung resections is cost-effective. J Thorac Dis 2018;10:5879-88.

40. Haro GJ, Sheu B, Marcus SG, et al. Perioperative Lung Resection Outcomes After Implementation of a Multidisciplinary, Evidence-based Thoracic ERAS Program. Ann Surg 2019. doi: 10.1097/ SLA.0000000000003719.

41. Huang H, Ma H, Chen S. Enhanced recovery after surgery using uniportal video-assisted thoracic surgery for lung cancer: A preliminary study. Thorac Cancer 2018;9:83-7.

42. Khandhar SJ, Schatz CL, Collins DT, et al. Thoracic enhanced recovery with ambulation after surgery: a 6-year experience. Eur J Cardiothorac Surg 2018;53:1192-8.

43. Li M, Ji H, Shi Y, et al. The efficacy of enhanced recovery after surgery for the perioperative care of lung cancer patients with thoracoscopy. Int J Clin Exp Med 2019;12:13855-62.

44. Lv H, Jiang X. Improvement effect of fast track surgery on lung cancer patients in perioperative period and influence on negative emotions. Int J Clin Exp Med 
2019;12:13601-9.

45. Madani A, Fiore JF, Wang Y, et al. An enhanced recovery pathway reduces duration of stay and complications after open pulmonary lobectomy. Surgery 2015;158:899-908.

46. Maruyama R, Miyake T, Kojo M, et al. Establishment of a clinical pathway as an effective tool to reduce hospitalization and charges after video-assisted thoracoscopic pulmonary resection. Jpn J Thorac Cardiovasc Surg 2006;54:387-90.

47. Numan RC, Klomp HM, Li W, et al. A clinical audit in a multidisciplinary care path for thoracic surgery: an instrument for continuous quality improvement. Lung Cancer 2012;78:270-5.

48. Rice D, Rodriguez-Restrepo A, Mena G, et al. Matched Pairs Comparison of an Enhanced Recovery Pathway Versus Conventional Management on Opioid Exposure and Pain Control in Patients Undergoing Lung Surgery. Ann Surg 2020. doi: 10.1097/SLA.0000000000003587.

49. Salati M, Brunelli A, Xiumè F, et al. Does fast-tracking increase the readmission rate after pulmonary resection? A case-matched study. Eur J Cardiothorac Surg 2012;41:1083-7.

50. Shiono S, Endo M, Suzuki K, et al. Impact of enhanced recovery after surgery on outcomes of elderly patients undergoing open thoracic surgery. Gen Thorac Cardiovasc Surg 2019;67:867-75.

51. Tahiri M, Goudie E, Jouquan A, et al. Enhanced recovery after video-assisted thoracoscopic surgery lobectomy: a prospective, historically controlled, propensity-matched clinical study. Can J Surg 2020;63:E233-40.

52. Van Haren RM, Mehran RJ, Mena GE, et al. Enhanced Recovery Decreases Pulmonary and Cardiac Complications After Thoracotomy for Lung Cancer. Ann Thorac Surg 2018;106:272-9.

53. Jin X, Liang Y, Zhou J, et al. Application of thoracoscopy with fast-track surgery in the thoracotomy of small-cell lung cancer. Biomedical Research (India) 2017;28:8473-6.

54. Walker WS, Leaver HA. Immunologic and stress responses following video-assisted thoracic surgery and open pulmonary lobectomy in early stage lung cancer.

Cite this article as: Li R, Wang K, Qu C, Qi W, Fang T, Yue $\mathrm{W}$, Tian $\mathrm{H}$. The effect of the enhanced recovery after surgery program on lung cancer surgery: a systematic review and metaanalysis. J Thorac Dis 2021;13(6):3566-3586. doi: 10.21037/jtd-21433
Thorac Surg Clin 2007;17:241-9, ix.

55. Kim JY, Sun V, Raz DJ, et al. The impact of lung cancer surgery on quality of life trajectories in patients and family caregivers. Lung Cancer 2016;101:35-9.

56. Carli F. Physiologic considerations of Enhanced Recovery After Surgery (ERAS) programs: implications of the stress response. Can J Anaesth 2015;62:110-9.

57. Leeman M, van Mil SR, Biter LU, et al. Reducing complication rates and hospital readmissions while revising the enhanced recovery after bariatric surgery (ERABS) protocol. Surg Endosc 2021;35:612-9.

58. Zhang L, Wang CC. Inflammatory response of macrophages in infection. Hepatobiliary Pancreat Dis Int 2014;13:138-52.

59. Alazawi W, Pirmadjid N, Lahiri R, et al. Inflammatory and Immune Responses to Surgery and Their Clinical Impact. Ann Surg 2016;264:73-80.

60. Oor JE, Daniels JM, Debets-Ossenkopp YJ, et al. Bronchial colonization and complications after lung cancer surgery. Langenbecks Arch Surg 2016;401:885-92.

61. Burke JR, Duarte IG, Thourani VH, et al. Preoperative risk assessment for marginal patients requiring pulmonary resection. Ann Thorac Surg 2003;76:1767-73.

62. Zhuang CL, Huang DD, Chen FF, et al. Laparoscopic versus open colorectal surgery within enhanced recovery after surgery programs: a systematic review and metaanalysis of randomized controlled trials. Surg Endosc 2015;29:2091-100.

63. Paul S, Altorki NK, Sheng S, et al. Thoracoscopic lobectomy is associated with lower morbidity than open lobectomy: a propensity-matched analysis from the STS database. J Thorac Cardiovasc Surg 2010;139:366-78.

64. Rogers LJ, Bleetman D, Messenger DE, et al. The impact of enhanced recovery after surgery (ERAS) protocol compliance on morbidity from resection for primary lung cancer. J Thorac Cardiovasc Surg 2018;155:1843-52.

65. Forster C, Doucet V, Perentes JY, et al. Impact of Compliance With Components of an ERAS Pathway on the Outcomes of Anatomic VATS Pulmonary Resections. J Cardiothorac Vasc Anesth 2020;34:1858-66. 


\section{Supplementary}

Table S1 Additional baseline characteristics of included studies

\begin{tabular}{|c|c|c|c|c|c|c|c|c|}
\hline \multirow{2}{*}{ Study (year) } & \multicolumn{2}{|c|}{ Mean BMI (kg/m²) } & \multicolumn{2}{|c|}{ FEV1 (\% predicted) } & \multicolumn{2}{|c|}{ Surgical procedures } & \multicolumn{2}{|c|}{ Extent of resection } \\
\hline & ERAS & Con. & ERAS & Con. & ERAS & Con. & ERAS & Con. \\
\hline Dong 2017 & $26.8[18.5-30.0]$ & $25.6[18.7-29.8]$ & $2.9[2.5-3.9]^{a}$ & $2.8[2.5-3.7]^{\mathrm{a}}$ & OPEN & OPEN & Pne & Pne \\
\hline Muehling 2008 & NR & NR & $2.05(0.68-4.5)^{a}$ & $2.4(1.4-2.8)^{a}$ & NR & NR & $\begin{aligned} \text { Lob }=20 ; \text { Pne } & =3 ; \text { Sle }=1 ; \text { Wed } \\
& =6\end{aligned}$ & 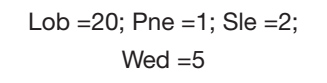 \\
\hline Boujibar 2018 & $26.6[23.7-30.4]$ & $25.0[22.5-30]$ & $61[54-67]$ & $61[58-71]$ & V/RATS & V/RATS & Lob & Lob \\
\hline Brunelli 2017 & $27.3[24-30]$ & 27 [24-30] & 89.2 [74-105] & $89.2[73-106]$ & VATS & VATS & Lob; Seg & Lob; Seg \\
\hline Chen 2020 & NR & NR & NR & NR & NR & NR & Lob & Lob \\
\hline Gao 2015 & NR & NR & NR & NR & $\begin{aligned} \text { OPEN } & =29 ; \text { VATS } \\
& =42\end{aligned}$ & $\begin{aligned} \text { OPEN } & =32 ; \text { VATS } \\
& =39\end{aligned}$ & Lob & Lob \\
\hline Glogowaska 2017 & NR & NR & $90[80-104]$ & 95 [83-101] & NR & NR & Lob $=61 ;$ Wed $=147$ Pne $=7$ & Lob $=46 ;$ Wed $=136$ Pne $=5$ \\
\hline Gonzalez 2018 & 24.9 (median) & 23.5 (median) & $92 \pm 18$ & $81 \pm 20$ & VATS & VATS & Lob =32; Seg =18 & Lob =30; Seg =20 \\
\hline Haro,G 2019 & NR & NR & $91[80-107]$ & 86 [72-100] & $\begin{array}{l}\text { OPEN }=47 ; \mathrm{V} / \\
\text { RATS }=79\end{array}$ & $\begin{array}{l}\text { OPEN }=102 ; \mathrm{V} / \\
\quad \text { RATS }=67\end{array}$ & Lob =62; Sublob =64 & Lob =81; Sublob =88 \\
\hline Huang 2018 & NR & NR & $94.09 \pm 10.09$ & $92.02 \pm 16.00$ & VATS & VATS & Lob; Wed & Lob; Wed \\
\hline Khandhar 2018 & NR & NR & NR & NR & VATS & VATS & Lob & Lob \\
\hline Li,M 2019 & NR & NR & $98.25 \pm 11.89$ & $97.89 \pm 11.44$ & VATS & VATS & NR & NR \\
\hline Lv,H 2019 & NR & NR & NR & NR & NR & NR & NR & $N R$ \\
\hline Madani 2015 & $27 \pm 6$ & $27 \pm 5$ & $87 \pm 24$ & $92 \pm 22$ & OPEN & OPEN & Lob & Lob \\
\hline Maruyama 2006 & NR & NR & NR & NR & VATS & VATS & $\begin{array}{c}\text { Lob =64; Seg+Wed =46; } \\
\text { Pne+Bilob =3 }\end{array}$ & $\begin{array}{c}\text { Lob =58; Seg+Wed =46; } \\
\text { Pne+Bilob =1 }\end{array}$ \\
\hline Numan RC 2012 & 25 [22-27] & 26 [23-28] & 95 [82-104] & 92 [77-101] & $\begin{aligned} \text { OPEN } & =50 ; \text { VATS } \\
& =25\end{aligned}$ & $\begin{aligned} \text { OPEN } & =79 ; \text { VATS } \\
& =15\end{aligned}$ & 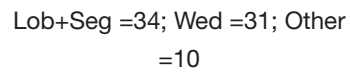 & $\begin{array}{c}\text { Lob+Seg }=42 ; \text { Wed =38; } \\
\text { Other }=14\end{array}$ \\
\hline Rice,D 2020 & NR & NR & NR & NR & $\begin{array}{c}\text { OPEN }=73 ; \mathrm{V} / \\
\text { RATS }=50\end{array}$ & $\begin{array}{c}\text { OPEN }=73 ; \mathrm{V} / \\
\text { RATS }=50\end{array}$ & $\begin{array}{c}\text { Wed+Seg =46; Lob =69; } \\
\text { Pne+Sle =8 }\end{array}$ & $\begin{array}{c}\text { Wed+Segm:46; Lob =69; } \\
\text { Pne+Sle =8 }\end{array}$ \\
\hline Salati 2012 & $26.3 \pm 4.8$ & $26.5 \pm 3.8$ & $84.6 \pm 18.5$ & $85.7 \pm 19$ & NR & NR & Lob & Lob \\
\hline Shiono 2019 & 22.8 [20.4-24.5] & 22.6 [20.9-25.] & $74[67.5-78.7]$ & 73.8 [68-80.5] & OPEN & OPEN & Lob =94; Seg =32 & Lob =100; Seg =26 \\
\hline Tahiri 2020 & $26.9 \pm 5.9$ & $27.5 \pm 5.6$ & $92.9 \pm 18.6$ & $86.8 \pm 22$ & VATS & VATS & Lob & Lob \\
\hline Van Haren 2018 & NR & NR & 86 [60-112] & 87 [62-112] & $\begin{array}{l}\text { OPEN }=166 ; \mathrm{V} / \\
\text { RATS }=176\end{array}$ & $\begin{array}{l}\text { OPEN }=1095 ; \mathrm{V} / \\
\text { RATS }=520\end{array}$ & $\begin{array}{c}\text { Lob =239; Pne }=15 ; \text { Sle =14; } \\
\text { Wed }=60 ; \text { Seg =32 }\end{array}$ & $\begin{array}{c}\text { Lob }=1202 ; \text { Pne }=91 ; \text { Sle =81; } \\
\quad \text { Wed }=237 ; \text { Seg }=125\end{array}$ \\
\hline
\end{tabular}

